Ergod. Th. of Dynam. Sys. (1985), 5, 565-586

Printed in Great Britain

\title{
On infinite tensor products of factors of type $\mathbf{I}_{2}$
}

\author{
T. GIORDANO AND G. SKANDALIS
}

Université de Genève, Section de Mathematiques, Case Postale 240, 2-4, rue du Lièvre, 1211 GENÈVE 24, Switzerland; Laboratoire de Mathematiques Fondamentales, UER 48, Université P. et M. Curie, 4 Place Jussieu, 75230 Paris Cedex 05, France

(Received 4 September 1984)

Abstract. It is proved, using Krieger's theorem, that ITPFI's of bounded type are ITPFI $_{2}$. This answers a question asked by E. J. Woods.

\section{0 . Introduction}

The main result of this paper asserts that every infinite tensor product of factors of type I (ITPFI) of bounded type is an ITPFI $_{2}$ factor (theorem 2.1). Its proof is based on Krieger's theorem ([12]).

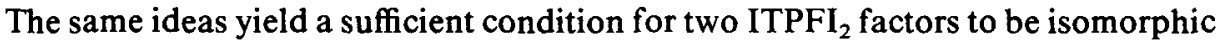
(theorem 3.1). This comes from Kakutani's criterion ([11]) for the equivalence of infinite product measures, applied in the context of the Connes-Takesaki flow of weights.

Theorem 3.1, in turn, allows us to sharpen theorem 2.1: namely, given an ITPFI factor of bounded type, we realize it explictly as an ITPFI ${ }_{2}$ in terms of eigenvalues and multiplicities (corollary 3.6). Together with a partial converse (proposition 4.4), theorem 3.1 gives also a rather surprising example (4.5).

In the proof of theorem 2.1, when Connes' invariant $T$ is $\{0\}$, we use the rather easy proposition 1.1. In the appendix we indicate the construction of the flow of weights used in this text. Theorem 2.1 solves question 4 of [16] and theorem 3.1 may be used to give an answer to question 7 of [16] (corollary 3.7). Note, finally, that theorem 2.1 admits a more elementary, but technical proof (remark 2.3).

We are very grateful to E. J. Woods who made this research possible by his kind invitation at Queen's University and also for many precious conversations and careful reading of the manuscript. We would also like to thank him with all the faculty and staff of the department of Mathematics and Statistics who contributed to make our stay as pleasant as possible-particularly Prof. U. Fixman, P. Ribenboim, E. Weimar-Woods, Dr. M. Khoshkam and also Z. Mansourati and J. Mináč.

The first author was financially supported in part by NSERC (Canada) and the Swiss National Fund for Scientific Research; the second author by NSERC (Canada). The authors wish to thank these institutions.

All the definitions and the notation can easily be found in the literature (for instance in [16]). However, we shall recall those definitions which are frequently used. 
Definitions (1) A factor $M$ is called ITPFI if it is of the form

$$
M=\bigotimes_{k=1}^{\infty}\left(M_{n_{k}}(C), \phi_{k}\right)
$$

acting on the Hilbert space $\bigotimes_{k=1}^{\infty}\left(H_{k}, \xi_{k}\right)$ where $M_{n_{k}}(C)$ denotes the algebra of $n_{k} \times n_{k}$ matrices acting on the Hilbert space $H_{k}\left(n_{k} \geq 2\right)$ and $\phi_{k}(x)=\left\langle x \xi_{k}, \xi_{k}\right\rangle$.

(2) If all the $n_{k}$ are bounded by a number $n, M$ is said to be an ITPFI of bounded type.

(3) If all the $n_{k}$ are equal to 2 (or to $m$ ) $M$ is said to be an ITPFI 2 (or ITPFI $I_{m}$ ).

Finally, if $M$ is a factor, $T(M)$ and $S(M)$ denote Connes' invariants $T$ and $S$ $([2, \S \S 1,3])$.

\section{A technical result}

Let $M$ be an ITPFI factor of bounded type. In order to prove that $M$ is an ITPFI 2 factor, we use, if $T(M)=\{0\}$, the following:

(1.1) Proposition. Let $M=\bigotimes_{k \geq 1}\left(M_{m+1}(C), \phi_{k}\right)$ be an $\operatorname{ITPFI}_{m+1}$, with $S(M) \subseteq$ $\{0,1\}$. Then, for every positive number $a$, there exist sequences $\left(\psi_{k}\right)_{k \geq 1}$ of states on $M_{m+1}(C)$ and $\left(\lambda_{p}\right)_{p \geq 0}$ of real numbers such that:

(i) the states $\bigotimes_{k \geq 1} \phi_{k}$ and $\bigotimes_{k \geq 1} \psi_{k}$ are weakly equivalent;

(ii) the eigenvalues of $\psi_{k}$ are $\left(1 / \Lambda_{k}^{\prime}, \eta_{k, 1} / \Lambda_{k}^{\prime}, \ldots, \eta_{k, m} / \Lambda_{k}^{\prime}\right.$ where $\eta_{k, j} \in\left\{\lambda_{p} ; p \geq 0\right\}$, $\left(\Lambda_{k}^{\prime}=1+\sum_{j=1}^{m} \eta_{k, j}\right) ;$ and

(iii) $\lambda_{0}=1$ and $e^{-(p+1) a}<\lambda_{p} \leq e^{-p a}$, for $p \geq 1$.

We write the eigenvalues of the state $\phi_{k}$ in the form $\left(\xi_{k, 0} / \Lambda_{k}, \xi_{k, 1} / \Lambda_{k}, \ldots, \xi_{k, m} / \Lambda_{k}\right)$, where $1=\xi_{k, 0} \geq \xi_{k, 1} \geq \cdots \geq \xi_{k, m}>0$ and $\Lambda_{k}=\sum_{i=0}^{m} \xi_{k, i}$.

We need the following application of lemma 8.6 of [1].

(1.2) LEMMA. Let a be a positive real number. If there exist disjoint subsets $Y, Z$ of $\mathbb{N} \times\{0, \ldots, m\}$ and a bijection $\alpha: Y \rightarrow Z$ such that $\sum_{(k, i) \in Y}\left|\xi_{k, i}^{\frac{1}{2}}-\xi_{\alpha(k, i)}^{\frac{1}{2}}\right|^{2}=\infty$ and $\left|\log \left(\xi_{a(k, i)} / \xi_{k, i}\right)\right| \leq a$, for all $(k, i) \in Y$, then $S(M) \cap\left[e^{-a}, 1\right) \neq \varnothing$.

Proof. Write $\alpha(k, i)=\left(l_{k, i}, j_{k, i}\right)$. Replacing if necessary the pair $\{(k, i), \alpha(k, i)\}$ by $\{\alpha(k, i),(k, i)\}$, we may assume that for all $(k, i) \in Y, l_{k, i} \geq k$. Put $\beta(k, i)=$ $\left|\xi_{k, i}^{k}-\xi_{\alpha(k, i)}^{\frac{1}{2}}\right|^{2},(k, i) \in Y$.

If $\sum_{(k, i) \in Y, l_{k, i}=k} \beta(k, i)=\infty$, then one may apply lemma 8.6 of [1] and get the conclusion (putting $K_{k}^{1}=\left\{\xi_{k, i} / \Lambda_{k} \mid(k, i) \in Y\right.$ and $\left.l_{k, i}=k\right\} ; \phi_{k}\left(\xi_{k, i} / \Lambda_{k}\right)=\xi_{\alpha(k, i)} / \Lambda_{k}$ if $\xi_{k, i} / \Lambda_{k} \in K_{k}^{1} ; K_{k}^{2}=\phi_{k}\left(K_{k}^{1}\right)$; note that $\left.\Lambda_{k} \leq m+1\right)$.

So, replacing $Y$ by $\left\{(k, i) \in Y \mid l_{k, i}>k\right\}$, we may furthermore assume that $l_{k, i}>k$, for all $(k, i) \in Y$.

Let $p: \mathbb{N} \times\{0, \ldots, m\} \rightarrow \mathbb{N}$ be the projection, $(p(k, i)=k)$. For $k \in p(Y)$, let $i_{k}$ be an element maximizing $\{\beta(k, i) \mid(k, i) \in Y\}$. We have

$$
\sum_{k \in p(Y)} \beta\left(k, i_{k}\right) \geq \frac{1}{m+1} \sum_{(k, i) \in Y} \beta(k, i)=+\infty .
$$


Replacing $Y$ by $\left\{\left(k, i_{k}\right) \mid k \in p(Y)\right\}$, we may now assume that the $\operatorname{map} p: Y \rightarrow \mathbb{N}$ is one-to-one. Maximizing, for a given $k, \beta\left(\alpha^{-1}(k, j)\right)$, we may also suppose that the map $p: Z \rightarrow \mathbb{N}$ is one-to-one.

Let $l: p(Y) \rightarrow p(Z)$ be given by $l(k)=l_{k, i_{k}}$. For $k \in p(Y)$, put

$$
r(k)= \begin{cases}0 & \text { if } k \notin p(Z), \\ \max \left\{i \mid k \in l^{i}(p(Y))\right\} & \text { if } k \in p(Z) .\end{cases}
$$

As $l(k)>k, \quad$ for all $k \in p(Y), \quad r(k) \leq k$. As $\sum_{k \in p(Y)} \beta\left(k, i_{k}\right)=\infty$, either $\sum_{r(k) \text { even }} \beta\left(k, i_{k}\right)=\infty$ or $\sum_{r(k) \text { odd }} \beta\left(k, i_{k}\right)=\infty$.

Assume, for instance, that $\sum_{k \in p(Y), r(k) \text { even }} \beta\left(k, i_{k}\right)=\infty$. Then, replacing $Y$ by $\left\{\left(k, i_{k}\right) \mid r(k)\right.$ even $\}$, the map $p: Y \cup Z \rightarrow \mathbb{N}$ is one-to-one. Write

$$
M=\left(\bigotimes_{k \in P(Y \cup Z)}\left(M_{m+1}, \phi_{k}\right)^{\prime}\right) \otimes\left(\bigotimes_{(k, i) \in Y}\left(M_{m+1} \otimes M_{m+1}, \phi_{k} \otimes \phi_{l(k)}\right)\right)
$$

and apply lemma 8.6 of [1], (putting

$$
K_{k, i}^{1}=\left\{\frac{\xi_{k, i} \xi_{l(k), 0}}{\Lambda_{k} \Lambda_{l(k)}}\right\}, \quad K_{k, i}^{2}=\left\{\frac{\xi_{k, 0} \xi_{\alpha(k, i)}}{\Lambda_{k} \Lambda_{l(k)}}\right\}
$$

and recalling that $\xi_{k, 0}=1$ for all $k$ and that $\left.\Lambda_{k} \leq m+1\right)$.

End of the proof of proposition 1.1. For $p \geq 0$, let

$$
E_{p}=\left\{(k, i) \in \mathbb{N} \times\{1, \ldots, m\} \mid \xi_{k, i} \in\left(e^{-(p-1) a}, e^{-p a}\right]\right\} .
$$

We have, by lemma $1.2, \sum_{(k, i) \in E_{p}}\left|\xi_{k, i}^{\frac{1}{2}}-1\right|^{2}<\infty$. Hence, for $p \neq 0$, \# $E_{p}$ is finite, where \# $E_{p}$ denotes the cardinality of $E_{p}$.

Choose $\lambda_{p} \in\left(e^{-(p+1) a}, e^{-p a}\right]$ such that

$$
\#\left\{(k, i) \in E_{p} \mid \xi_{k, i}<\lambda_{p}\right\} \leq \#\left\{(k, i) \in E_{p} \mid \xi_{k, i} \geq \lambda_{p}\right\}
$$

and

$$
\#\left\{(k, i) \in E_{p} \mid \xi_{k, i}>\lambda_{p}\right\} \leq \#\left\{(k, i) \in E_{p} \mid \xi_{k, i} \leq \lambda_{p}\right\} .
$$

Let $F_{p}$ and $G_{p}$ be disjoint sets with the same cardinality, such that

$$
F_{p} \supseteq\left\{(k, i) \in E_{p} \mid \xi_{k, i}<\lambda_{p}\right\}
$$

and

$$
G_{p} \supseteq\left\{(k, i) \in E_{p} \mid \xi_{k, i}>\lambda_{p}\right\}
$$

and let $\alpha_{p}: F_{p} \rightarrow G_{p}$ be a bijection.

If $(k, i) \in F_{p}$, we have

Therefore

$$
\begin{aligned}
\left|\xi_{k, i}^{\frac{1}{2}}-\xi_{\alpha_{p}(k, i)}^{\frac{1}{2}}\right|^{2} & =\left|\lambda_{p}^{\frac{1}{2}}-\xi_{k, i}^{\frac{1}{2}}+\xi_{\alpha_{p}(k, i)}^{\frac{1}{2}}-\lambda_{p}^{\frac{1}{2}}\right|^{2} \\
& \geq\left|\lambda_{p}^{\frac{1}{2}}-\xi \xi_{, i}\right|^{2}+\left|\xi_{\alpha_{p}(k, i)}^{\frac{1}{2}}-\lambda_{p}^{\frac{1}{2}}\right|^{2} .
\end{aligned}
$$

$$
\sum_{(k, i) \in F_{p}}\left|\xi_{k, i}^{k}-\xi_{\alpha_{p}(k, i)}^{\frac{1}{2}}\right|^{2} \geq \sum_{(k, i) \in E_{p}}\left|\xi_{k, i}^{\frac{1}{2}}-\lambda_{p}^{\frac{1}{2}}\right|^{2}
$$


We get, using lemma 1.2,

$$
\sum_{p=1}^{\infty} \sum_{(k, i) \in E_{p}}\left|\xi_{k, i}^{\frac{1}{k}}-\lambda_{p}^{\frac{1}{2}}\right|^{2} \leq \sum_{p=1}^{\infty} \sum_{(k, i) \in F_{p}}\left|\xi_{k, i}^{\frac{1}{k}}-\xi_{\alpha_{p}(k, i)}^{\frac{1}{2}}\right|^{2}<\infty
$$

Put $\lambda_{0}=1$. If $(k, i) \in E_{p}, p \in \mathbb{N}$, put $\eta_{k, i}=\lambda_{p}$. As $\sum_{(k, i) \in E_{0}}\left|\xi_{k, i}^{l}-1\right|^{2}<\infty$, we get $\sum_{(k, i) \in E_{0}}\left|\xi_{k, i}^{\frac{1}{2}}-\eta_{k, i}^{\frac{1}{2}}\right|^{2}<\infty$. Put $\Lambda_{k}^{\prime}=\sum_{i=0}^{m} \eta_{k, i},\left(\eta_{k, 0}=1\right)$.

Note that if $x$ and $y$ are non-zero vectors of a normed vector space, then we have

Therefore,

$$
\begin{aligned}
\left\|\frac{x}{\|x\|}-\frac{y}{\|y\|}\right\| & \leq \frac{1}{\|x\|}\left(\|x-y\|+\left\|y\left(1-\frac{\|x\|}{\|y\|}\right)\right\|\right) \\
& \leq \frac{1}{\|x\|}(\|x-y\|+|\|y\|-\|x\||) \\
& \leq \frac{2}{\|x\|}\|x-y\| .
\end{aligned}
$$

Hence

$$
\begin{aligned}
\sum_{i=0}^{m}\left|\left(\frac{\xi_{k, i}}{\Lambda_{k}}\right)^{\frac{1}{2}}-\left(\frac{\eta_{k, i}}{\Lambda_{k}^{\prime}}\right)^{\frac{1}{2}}\right|^{2} & \leq \frac{4}{\Lambda_{k}} \sum_{i=0}^{m}\left|\xi_{k, i}^{\frac{1}{k}}-\eta_{k}^{\frac{1}{k}}\right|^{2} \\
& \leq 4 \sum_{i=0}^{m}\left|\xi_{k, i}^{\frac{1}{2}}-\eta_{k, i}^{\frac{1}{2}}\right|^{2}
\end{aligned}
$$

$$
\sum_{(k, i)}\left|\left(\frac{\xi_{k, i}}{\Lambda_{k}}\right)^{\frac{1}{2}}-\left(\frac{\eta_{k, i}}{\Lambda_{k}^{\prime}}\right)^{\frac{1}{2}}\right|^{2}<\infty .
$$

This inequality gives the weak equivalence of $\otimes_{k \geq 1} \phi_{k}$ and $\bigotimes_{k \geq 1} * \psi_{k}$.

2. Infinite tensor products of bounded type

In this section, we prove

(2.1) THEOREM. Every ITPFI factor of bounded type is (isomorphic to) an ITPFI $_{2}$ factor.

This answers a question of E. J. Woods [16, $4 \S 6])$.

Up to isomorphism, there exists a unique ITPFI factor, in each of the cases I, $\mathrm{II}_{1}, \mathrm{II}_{\infty}$ and $\mathrm{III}_{\lambda}, \lambda \in(0,1]$ and it can be realized as an ITPFI ${ }_{2}([1])$. Therefore, the only interesting case of theorem 2.1 is the type $\mathrm{III}_{0}$ case.

An ITPFI factor of bounded type is a finite tensor product of homogeneous ITPFI factors.

We proceed by induction and show that every $\operatorname{ITPFI}_{m+2}$ factor $M(m \geq 1)$ is a tensor product of an ITPFI $I_{m+1}$ factor by an ITPFI $_{2}$ factor.

Therefore, let $M=\bigotimes_{k \geq 1}\left(M_{m+2}(C), \phi_{k}\right)$ be an ITPFI $I_{m+2}$ factor of type III $_{0}$. We write the eigenvalues of the state $\phi_{k}$ in the form $\left(\xi_{k, 0} / \Lambda_{k}, \xi_{k, 1} / \Lambda_{k}, \ldots, \xi_{k, m} / \Lambda_{k}\right.$, $\left.\xi_{k, m+1} / \Lambda_{k}\right)$ where $1=\xi_{k, 0} \geq \xi_{k, 1} \geq \cdots \geq \xi_{k, m} \geq \xi_{k, m+1}>0$ and $\Lambda_{k}=\sum_{i=0}^{m+1} \xi_{k, i}$

If $T(M) \neq\{0\}$, we might assume that all the $\xi_{k, i}$ are of the form $\lambda^{p}$, where $\lambda \in(0,1)$ is such that $2 \pi / \log \lambda \in T(M)$. In order to treat the case $T(M)=\{0\}$, we use

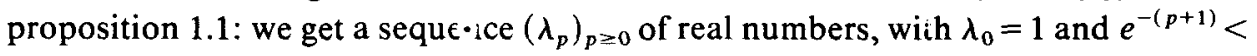
$\lambda_{p} \leq e^{-p}$, for all $p$; we suppose that the $\xi_{k, i}$ 's are chosen in the sequence $\left(\lambda_{p}\right)_{p \geq 0}$. 
Gathering the terms which have the same eigenvalue list, we may write:

$$
M=\bigotimes_{p, q}\left(\left(M_{m+2}(C), \phi_{p, q}\right)^{\otimes L_{p, q}}\right),
$$

where $p=\left(p_{1}, \ldots, p_{m}\right) ;(p, q)$ runs over all the $(m+1)$-tuples $0 \leq p_{1} \leq \cdots \leq p_{m} \leq q$ and $\phi_{p, q}$ is a state with eigenvalues $\left(1 / \Lambda_{p, q}, \lambda_{p 1} / \Lambda_{p, q}, \ldots, \lambda_{p_{m}} / \Lambda_{p, q}, \lambda_{q} / \Lambda_{p, q}\right), \Lambda_{p, q}=$ $\Lambda_{p}+\lambda_{q}$, where $\Lambda_{p}=1+\sum_{i=1}^{m} \lambda_{p_{i}}$

As $M$ is of type $\operatorname{III}_{0}, L_{p, q}<\infty$ for $(p, q) \neq(0,0)$. Sin.e $M$ is isomorphic to $M \otimes R$ ( $R$ is the hyperfinite factor of type $\mathrm{II}_{1}$ ), we may assume that $L_{0,0}=0$.

For all $(p, q)$ define integers $K_{p, q}$ and $J_{p, q}$ by

$$
K_{p, q}=L_{p, q}-\left[\frac{\lambda_{q} L_{p, q}}{\Lambda_{p, q}}\right] \text { and } J_{p, q}=\left[\frac{L_{p, q}\left(1+\lambda_{q}\right)}{\Lambda_{p, q}}\right]
$$

where if $x \in \mathbb{R},[x]$ denotes its integral part.

Let $\phi_{p}^{\prime}$ (resp. $\left.\phi_{q}^{\prime \prime}\right)$ be a stäe on $M_{m+1}(C)$ (resp. $\left.M_{2}(C)\right)$ with eigenvalues $\left(1 / \Lambda_{p}\right.$, $\left.\lambda_{p_{1}} / \Lambda_{p}, \ldots, \lambda_{p_{m}} / \Lambda_{p}\right)$, (resp. $\left.\left(1 / 1+\lambda_{q}\right), \lambda_{q} /\left(1+\lambda_{q}\right)\right)$. Set

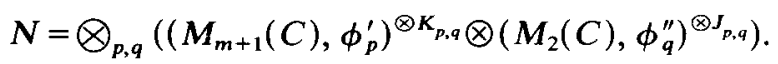

We want to prove that $M$ is isomorphic to $N$. by

For all $(p, q)$, consider the (probability) measures $\mu_{p, q}$ and $\mu_{p, q}^{\prime}$ on $\mathbb{N}^{m} \times \mathbb{N}$ given

$$
\mu_{p, q}(k, j)=\frac{L_{p, q} !}{k ! j !\left(L_{p, q}-|k|-j\right) !} \cdot \frac{\lambda_{p}^{k} \lambda_{q}^{j}}{\Lambda_{p, q}^{L_{p q}}},
$$

where $\mu_{p, q}(k, j)=0$ if $|k|+j>L_{p, q}$, and $k !=\prod_{i=1}^{m} k_{i} ! ;|k|=\sum_{i=1}^{m} k_{i} ; \lambda_{p}^{k}=\prod_{i=1}^{m} \lambda_{p_{i}}^{k_{i}}$;

$$
\mu_{p, q}^{\prime}(k, j)=\frac{K_{p, q} !}{k !\left(K_{p, q}-|k|\right) !} \cdot \frac{J_{p, q} !}{j !\left(J_{p, q}-j\right) !} \cdot \frac{\lambda_{p}^{k} \lambda_{q}^{j}}{\Lambda_{p}^{K_{p, q}}\left(1+\lambda_{q}\right)^{J_{p, q}}},
$$

with $\mu_{p, q}^{\prime}(k, j)=0$ if $|k|>K_{p, q}$ or $j>J_{p, q}$.

Let $(\Omega, \nu)$, (resp. $\left.\left(\Omega, \nu^{\prime}\right)\right)$ be the product measure space $(\Omega, \nu)=\prod_{(p, q)}\left(\mathbb{N}^{m} \times \mathbb{N}\right.$, $\left.\mu_{p, q}\right)\left(\right.$ resp. $\left.\left(\Omega, \nu^{\prime}\right)=\prod_{(p, q)}\left(\mathbb{N}^{m} \times \mathbb{N}, \mu_{p, q}^{\prime}\right)\right)$. Let $B \subseteq \Omega$, with $\nu(B) \neq 0$ and $\nu^{\prime}(B) \neq 0$.

Let $\mathscr{R}$ be the equivalence relation on $B \times \mathbb{R}$ given by: $(x, t) \mathscr{R}(y, s)$ iff there exists a finite subset $E$ of indices $(p, q)$ such that $x_{p, q}=y_{p, q}$, for all $(p, q) \notin E$ and

$$
\sum_{(p, q) \in E} T_{p, q}\left(x_{p, q}\right)+t=\sum_{(p, q) \in E} T_{p, q}\left(y_{p, q}\right)+s
$$

where $T_{p, q}(k, j)=-\sum_{i=1}^{m} k_{i} \log \lambda_{p_{i}}-j \log \lambda_{q}$, for $(k, j)=\left(k_{1}, k_{2}, \ldots, k_{m}, j\right) \in \mathbb{N}^{m} \times \mathbb{N}$.

The flow of weights of $M$ (resp. $N$ ) is given by the action of $\mathbb{R}$ by translation on $(B \times \mathbb{R}, \nu \times d x) / \mathscr{R}$ (resp. $\left.\left(B \times \mathbb{R}, \nu^{\prime} \times d x\right) / \mathscr{R}\right)$ (see appendix).

Using Krieger's theorem ([12]) to prove the isomorphism between $M$ and $N$, it is enough to show that the measures $\nu$ and $\nu^{\prime}$ are not (mutually) singular (i.e. equivalent on a subset $B \subseteq \Omega$ ). This is done using Kakutani's criterion on infinite product measures $([11$, p. 453$])$ and the following lemma.

(2.2) LEMMA. Let $1 \geq \xi_{1} \geq \xi_{2} \geq \cdots \geq \xi_{m} \geq \lambda>0$ be real numbers and let $L$ be a positive integer. Let $\mu$ and $\mu^{\prime}$ be the (probability) measures on $\mathbb{N}^{m} \times \mathbb{N}$ given by

$$
\begin{aligned}
& \mu(k, j)=\frac{L !}{k ! j !(L-|k|-j) !} \frac{\xi^{k} \lambda^{j}}{\Lambda^{L}}, \\
& (\mu(k, j)=0 \text { if }|k|+j>L) ;
\end{aligned}
$$




$$
\mu^{\prime}(k, j)=\frac{K !}{k !(K-|k|) !} \cdot \frac{J !}{j !(J-j) !} \cdot \frac{\xi^{k} \lambda^{j}}{(\Lambda-\lambda)^{K}(1+\lambda)^{j}},
$$

$\left(\mu^{\prime}(k, j)=0\right.$ if $|k|>K$ or $\left.j>J\right)$; where $\Lambda=1+\sum_{i=1}^{m} \xi_{i}+\lambda, K=L-[L \lambda / \Lambda], J=$ $[L(1+\lambda) / \Lambda],\left(\xi^{k}=\prod_{i=1}^{m} \xi_{i}^{k_{i}}\right)$. Then

$$
\rho\left(\mu, \mu^{\prime}\right)=\sum_{(k, j)} \mu(k, j)^{\frac{1}{2}} \mu^{\prime}(k, j)^{\frac{1}{2}} \geq 1-2 \lambda .
$$

Proof. Let $\alpha$ and $\alpha^{\prime}$ be the probability distributions of the random variable $j$, with respect to $\mu$ and $\mu^{\prime}$. They are measures on $\mathbb{N}$, given by

$$
\alpha(j)=\sum_{k} \mu(k, j)=\frac{L !}{j !(L-j) !} \frac{\lambda^{j}(\Lambda-\lambda)^{L-j}}{\Lambda^{L}}
$$

and

$$
\alpha^{\prime}(j)=\sum_{k} \mu^{\prime}(k, j)=\frac{J !}{j !(J-j) !} \frac{\lambda^{j}}{(1+\lambda)^{J}} .
$$

Let $\beta_{j}$ and $\beta^{\prime}$ be the conditional probabilities of the random variable $k$, given $j$. ( $\beta^{\prime}$ does not depend on $j$, as the random variables $j$ and $k$ are independent for $\mu^{\prime}$.) They are measures on $\mathbb{N}^{m}$ given by

$$
\begin{aligned}
& \beta_{j}(k)=\frac{\mu(k, j)}{\alpha(j)}=\frac{(L-j) !}{k !(L-j-|k|) !} \frac{\xi^{k}}{(\Lambda-\lambda)^{L-j}}, \\
& \beta^{\prime}(k)=\frac{\mu^{\prime}(k, j)}{\alpha^{\prime}(j)}=\frac{K !}{k !(K-|k|) !} \frac{\xi^{k}}{(\Lambda-\lambda)^{K}} .
\end{aligned}
$$

We have:

$$
\begin{aligned}
\rho\left(\mu, \mu^{\prime}\right) & =\sum_{k, j} \alpha(j)^{\frac{1}{2}} \beta_{j}(k)^{\frac{1}{2}} \alpha^{\prime}(j)^{\frac{1}{2}} \beta^{\prime}(k)^{\frac{1}{2}} \\
& =\sum_{j} \alpha(j)^{\frac{1}{2}} \alpha^{\prime}(j)^{\frac{1}{2}} \sum_{k} \beta_{j}(k)^{\frac{1}{2}} \beta^{\prime}(k)^{\frac{1}{2}} \\
& =\sum_{j} \alpha(j)^{\frac{1}{2}} \alpha^{\prime}(j)^{\frac{1}{2}} \rho\left(\beta_{j}, \beta^{\prime}\right) \\
& =\rho\left(\alpha, \alpha^{\prime}\right)-\sum_{j} \alpha(j)^{\frac{1}{2}} \alpha^{\prime}(j)^{\frac{1}{2}}\left(1-\rho\left(\beta_{j}, \beta^{\prime}\right)\right) .
\end{aligned}
$$

As $1-\rho\left(\beta_{j}, \beta^{\prime}\right) \geq 0$ and $\frac{1}{2}\left(\alpha(j)+\alpha^{\prime}(j)\right) \geq \alpha(j)^{\frac{1}{2}} \alpha^{\prime}(j)^{\frac{1}{2}}$,

$$
\rho\left(\mu, \mu^{\prime}\right) \geq \rho\left(\alpha, \alpha^{\prime}\right)-\frac{1}{2} \sum_{j}\left(\alpha(j)+\alpha^{\prime}(j)\right)\left(1-\rho\left(\beta_{j}, \beta^{\prime}\right)\right) .
$$

Therefore, we need only to estimate $\rho\left(\alpha, \alpha^{\prime}\right)$ and the expectations with respect to $\alpha$ and $\alpha^{\prime}$ of $1-\rho\left(\beta_{j}, \beta^{\prime}\right)$.

(a) Computation of $\rho\left(\beta_{j}, \beta^{\prime}\right)$. Let $K_{1}=\inf (K, L-j)$ and $K_{2}=\sup (K, L-j)$. If $K \geq L-j$, put $\gamma_{1}=\beta_{j}$ and $\gamma_{2}=\beta^{\prime}$; if $K<L-j$, put $\gamma_{1}=\beta^{\prime}$ and $\gamma_{2}=\beta_{j}$. We get:

$$
\frac{\gamma_{2}(k)}{\gamma_{1}(k)}=\frac{K_{2} !\left(K_{1}-|k|\right) !}{K_{1} !\left(K_{2}-|k|\right) !} \cdot \frac{1}{(\Lambda-\lambda)^{K_{2}-K_{1}}}=\prod_{i=1}^{K_{2}-K_{1}} \frac{K_{1}+i}{\left(K_{1}-|k|+i\right)(\Lambda-\lambda)} .
$$

Using the inequality $\log (a / b) \geq(a-b) / a$, we get

$$
\log \frac{\gamma_{2}(k)}{\gamma_{1}(k)} \geq \sum_{i=1}^{K_{2}-K_{1}} \frac{|k|(\Lambda-\lambda)-(\Lambda-\lambda-1)\left(K_{1}+i\right)}{K_{1}+i}
$$


Hence,

$$
\left(\frac{\gamma_{2}(k)}{\gamma_{1}(k)}\right)^{\frac{1}{2}} \geq 1+\frac{1}{2} \sum_{i=1}^{K_{2}-K_{1}} \frac{|k|(\Lambda-\lambda)-(\Lambda-\lambda-1)\left(K_{1}+i\right)}{K_{1}+i} .
$$

Now, $\rho\left(\gamma_{1}, \gamma_{2}\right)=\sum_{k} \gamma_{1}(k)\left(\gamma_{2}(k) / \gamma_{1}(k)\right)^{\frac{1}{2}}$. As $\sum_{k}|k| \gamma_{1}(k)=K_{1}(\Lambda-\lambda-1) /(\Lambda-\lambda)$, we get

$$
\rho\left(\gamma_{1}, \gamma_{2}\right) \geq 1-\frac{1}{2} \sum_{i=1}^{K_{2}-K_{1}} \frac{(\Lambda-\lambda-1) i}{K_{1}+i}
$$

As for $i \leq K_{2}-K_{1}, i /\left(K_{1}+i\right) \leq\left(K_{2}-K_{1}\right) / K_{2}$, we derive

$$
\rho\left(\gamma_{1}, \gamma_{2}\right) \geq 1-\frac{\left(K_{2}-K_{1}\right)^{2}}{2 K_{2}}(\Lambda-\lambda-1)
$$

Hence, $\rho\left(\beta_{j}, \beta^{\prime}\right) \geq 1-\frac{(K-L+j)^{2}}{2 K}(\Lambda-\lambda-1)$. Therefore

$$
\sum_{j} \alpha(j)\left(1-\rho\left(\beta_{j}, \beta^{\prime}\right)\right) \leq \frac{\Lambda-\lambda-1}{2 K} \sum_{j} \alpha(j)\left(\left(j-\frac{\lambda L}{\Lambda}\right)+\left(\frac{\lambda L}{\Lambda}+K-L\right)\right)^{2} .
$$

As the expectation $E_{\alpha}(j)$ of $j$, with respect to $\alpha$, is $L \lambda / \Lambda$ and its variance $\sigma_{\alpha}^{2}(j)$ is $L \lambda(\Lambda-\lambda) / \Lambda^{2}$, we get:

$$
\sum_{j} \alpha(j)\left(1-\rho\left(\beta_{j}, \beta^{\prime}\right)\right) \leq \frac{\Lambda-\lambda-1}{2 K}\left(\frac{L \lambda(\Lambda-\lambda)}{\Lambda^{2}}+\left(\frac{\lambda L}{\Lambda}+K-L\right)^{2}\right) .
$$

As $E_{\alpha^{\prime}}(j)=\lambda J / 1+\lambda$ and $\sigma_{\alpha^{\prime}}^{2}(j)=\lambda J /(1+\lambda)^{2}$, we get in the same way:

$$
\sum_{j} \alpha^{\prime}(j)\left(1-\rho\left(\beta_{j}, \beta^{\prime}\right)\right) \leq \frac{\Lambda-\lambda-1}{2 K}\left(\frac{\lambda J}{(1+\lambda)^{2}}+\left(\frac{\lambda J}{1+\lambda}+K-L\right)^{2}\right) .
$$

As by the definition of $K, L(\Lambda-\lambda) / K \Lambda \leq 1$ and, by the definition of $J, J /(1+\lambda) \leq$ $L / \Lambda \leq K /(\Lambda-\lambda)$, we get:

$$
\left(\frac{\Lambda-\lambda-1}{2 K}\right) \frac{L \lambda(\Lambda-\lambda)}{\Lambda^{2}} \leq \frac{\lambda}{2} \frac{\Lambda-\lambda-1}{\Lambda} \leq \frac{\lambda}{2}
$$

and

$$
\left(\frac{\Lambda-\lambda-1}{2 K}\right) \frac{\lambda J}{(1+\lambda)^{2}} \leq \frac{\lambda}{2} \frac{\Lambda-\lambda-1}{(\Lambda-\lambda)(1+\lambda)} \leq \frac{\lambda}{2} .
$$

If $L \lambda / \Lambda<1$, then $K=L$ and $(\lambda J)^{2} /(1+\lambda)^{2} \leq(\lambda L / \Lambda)^{2} \leq \lambda L / \Lambda$. If $L \lambda / \Lambda \geq 1$, then $0 \leq(\lambda L / \Lambda)+K-L=K-((L-(\lambda L / \Lambda))<1$, by the definition of $K$. Also, by the definition of $J, \lambda J /(1+\lambda) \leq \lambda L / \Lambda(1+\lambda) \leq \lambda L / \Lambda$; thus,

$$
-1 \leq \frac{-\lambda}{1+\lambda}<\frac{\lambda J}{1+\lambda}+K-L \leq \frac{\lambda L}{\Lambda}+K-L<1 .
$$

In all cases,

$$
\left(\frac{\lambda L}{\Lambda}+K-L\right)^{2} \leq \frac{\lambda L}{\Lambda} \text { and }\left(\frac{\lambda J}{1+\lambda}+K-L\right)^{2} \leq \frac{\lambda L}{\Lambda}
$$

Hence, we get:

$$
\sum_{j}\left(\alpha(j)+\alpha^{\prime}(j)\right)\left(1-\rho\left(\beta_{j}, \beta^{\prime}\right)\right) \leq \frac{\lambda}{2}+\frac{\Lambda-\lambda-1}{2 K} \cdot \frac{L \lambda}{\Lambda}+\frac{\lambda}{2}+\frac{\Lambda-\lambda-1}{2 K} \cdot \frac{L \lambda}{\Lambda} \leq 2 \lambda .
$$


(b) Computation of $\rho\left(\alpha, \alpha^{\prime}\right)$.

$$
\frac{\alpha(j)}{\alpha^{\prime}(j)}=\frac{L !(J-j) !(1+\lambda)^{J}}{J !(L-j) ! \Lambda^{L}} \cdot(\Lambda-\lambda)^{L-j}=\left(\prod_{i=1}^{L-J} \frac{J+i}{J-j+i}\right) \frac{(1+\lambda)^{J}(\Lambda-\lambda)^{L-j}}{\Lambda^{L}} .
$$

Let $e=E_{\alpha^{\prime}}(j)=J \lambda /(1+\lambda)$. Put

We have

$$
V_{e}=\left(\prod_{i=1}^{L-J} \frac{J+i}{J-e+i}\right) \frac{(1+\lambda)^{J}(\Lambda-\lambda)^{L-e}}{\Lambda^{L}}
$$

$$
\frac{\alpha(j)}{\alpha^{\prime}(j)}=\left(\prod_{i=1}^{L-J} \frac{J-e+i}{J-j+i}\right)(\Lambda-\lambda)^{e-j} V_{e^{*}}
$$

As $\log (a / b) \geq(a-b) / a$, we get

Hence,

$$
\log \frac{\alpha(j)}{\alpha^{\prime}(j)} \geq \log V_{e}+(e-j) \log (\Lambda-\lambda)+\sum_{i=1}^{L-J} \frac{j-e}{J-e+i}
$$

Therefore,

$$
\left(\frac{\alpha(j)}{\alpha^{\prime}(j)}\right)^{\frac{1}{2}} \geq V_{e}^{\frac{1}{2}}\left[1+\frac{1}{2}(e-j)\left(\log (\Lambda-\lambda)-\sum_{i=1}^{L-J} \frac{1}{J-e+i}\right)\right] .
$$

as $\sum_{j}(e-j) \alpha^{\prime}(j)=0$.

$$
\rho\left(\alpha, \alpha^{\prime}\right)=\sum_{j}\left(\frac{\alpha(j)}{\alpha^{\prime}(j)}\right)^{\frac{1}{2}} \alpha^{\prime}(j) \geq V_{e}^{\frac{1}{2}},
$$

Now,

$$
V_{e}=\frac{L}{L-e} \frac{J-e}{J}\left(\prod_{i=0}^{L-J-1} \frac{J+i}{J-e+i}\right) \frac{(1+\lambda)^{J}(\Lambda-\lambda)^{L-e}}{\Lambda^{L}}
$$

As $\log (J+t) /(J-e+t)$ is a decreasing function of $t \geq 0$, we get

$$
\begin{aligned}
\sum_{i=0}^{L-J-1} \log \frac{J+i}{J-e+i} & \geq \int_{0}^{L-J}[\log (J+t)-\log (J-e+t)] d t \\
& \geq L \log L-J \log J-(L-e) \log (L-e)+(J-e) \log (J-e)
\end{aligned}
$$

Hence,

$$
\begin{aligned}
V_{e} & \geq \frac{J-e}{J} \cdot \frac{L^{L}(J-e)^{(J-e)}}{(L-e)^{L-e} J^{J}} \cdot \frac{(1+\lambda)^{J}(\Lambda-\lambda)^{L-e}}{\Lambda^{L}}=\frac{J-e}{J} \cdot \frac{\left(\frac{L}{\Lambda}\right)^{L}(J-e)^{J-e}}{\left(\frac{L-e}{\Lambda-\lambda}\right)^{(L-e)}\left(\frac{J}{1+\lambda}\right)^{J}} \\
& \geq \frac{J-e}{J} \cdot \frac{\left(\frac{L}{\Lambda(J-e)}\right)^{L}}{\left(\frac{L-e}{(\Lambda-\lambda)(J-e)}\right)^{L-e}} \cdot \frac{1}{\left(\frac{J}{(J-e)(1+\lambda)}\right)^{J}}
\end{aligned}
$$

Now, by definition of $e, J=(J-e)(1+\lambda)$. Put $\varepsilon=(L(1+\lambda) / \Lambda)-J$; (we have $0 \leq \varepsilon<1$ ). We get

$$
V_{e} \geq \frac{1}{1+\lambda} \cdot \frac{\left(1+\frac{\varepsilon}{J}\right)^{L}}{\left(1+\frac{\varepsilon \Lambda}{(\Lambda-\lambda) J}\right)^{L-e}} \geq \frac{1}{1+\lambda}\left(\frac{\left(1+\frac{\varepsilon}{J}\right)}{1+\frac{\varepsilon \Lambda}{(\Lambda-\lambda) J}}\right)^{L-e}
$$


But,

$$
\begin{aligned}
(L-e) \log \left(\frac{1+\frac{\varepsilon}{J}}{1+\frac{\varepsilon \Lambda}{(\Lambda-\lambda) J}}\right) & \geq(L-e) \frac{\frac{\varepsilon}{J}\left(1-\frac{\Lambda}{\Lambda-\lambda}\right)}{1+\frac{\varepsilon}{J}}=-\frac{(L-e) \varepsilon \lambda}{(\varepsilon+J)(\Lambda-\lambda)} \\
& \geq-\frac{\lambda \Lambda(L-e) \varepsilon}{L(1+\lambda)(\Lambda-\lambda)} \geq-\lambda,
\end{aligned}
$$

as $L-e \leq L, \varepsilon<1$ and $\Lambda \leq(1+\lambda)(\Lambda-\lambda)$. Hence,

$$
V_{e} \geq \frac{1}{1+\lambda} \exp (-\lambda)>(1-\lambda)^{2}
$$

Thus,

$$
\rho\left(\alpha, \alpha^{\prime}\right) \geq V_{e}^{\frac{1}{2}}>1-\lambda .
$$

By (1), (2) and (3), we get the result.

End of the proof of theorem 2.1. For a given $q$, there are $(q+m) ! / q ! m ! \leq(q+1)^{m}$ possible choices of $(p, q)$. As $\sum_{p, q} \lambda_{q} \leq \sum_{q}(q+1)^{m} \lambda_{q} \leq \sum_{q}(q+1)^{m} e^{--q}<\infty$, we get $\prod_{(p, q)} \rho\left(\mu_{p, q}, \mu_{p, q}^{\prime}\right) \neq 0$. By Kakutani's criterion, ([11, pp. 453-455]), the product measures $\nu$ and $\nu^{\prime}$ are not singular. The proof is now complete.

$N . B$. We actually used a straightforward consequence of Kakutani's criterion: though neither $\mu_{p, q}$, nor $\mu_{p, q}^{\prime}$ is absolutely continuous with respect to the other, as $\prod_{(p, q)} \rho\left(\mu_{p, q}, \mu_{p, q}^{\prime}\right) \neq 0$, the product measures $\nu$ and $\nu^{\prime}$ are not singular. (cf. [11, remark 22.37 , p. 455$]$.

(2.3) Remark. We can prove theorem 2.1 in a more computational way but without using Krieger's theorem. We need (keeping the above notation) the following:

Proposition. There exist:

(i) projections $P_{p, q}$ in $A_{p, q}=\left(M_{m+2}(C)\right)^{\otimes L_{p, q}}$ and $Q_{p, q}$ in $B_{p, q}=\left(M_{m+1}(C)^{\otimes K_{p, q} \otimes}\right.$ $\left(M_{2}(C)\right)^{\otimes J_{p, q}}$;

(ii) states $\chi_{p, q}$ on matrix algebras $C_{p, q}$ and integers $r_{p, q}, r_{p, q}^{\prime}$; such that

(a) $\prod_{p, q} \Phi_{p, q}\left(P_{p, q}\right)>0, \quad \prod_{p, q} \Psi_{p, q}\left(Q_{p, q}\right)>0$, where $\Phi_{p, q}=\phi_{p, q}^{\otimes L_{p, q}}$ and $\Psi_{p, q}=$

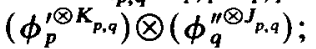

(b) $\left(C_{p, q} ; \chi_{p, q}\right) \otimes\left(M_{r_{p, q}}(C), \operatorname{Tr}\right)$ is isomorphic to $\left(A_{p, q}, \Phi_{p, q}\right)$ reduced by $P_{p, q}$, and $\left(C_{p, q}, \chi_{p, q}\right) \otimes\left(M_{r_{p, q}^{\prime}}(C), \mathrm{Tr}\right)$ is isomorphic to $\left(B_{p, q}, \Psi_{p, q}\right)$, reduced by $Q_{p, q}$

Then, the projections $P=\bigotimes_{p, q} P_{p, q} \in M$ and $Q=\bigotimes_{p, q} Q_{p, q} \in N$ are non-zero, and $M_{p} \otimes R \cong N_{Q} \otimes R$, where $R$ denotes the hyperfinite factor of type $\mathrm{II}_{1}$.

As $M$ is of type III, $M$ and $N$ are isomorphic. $\left(M \cong M_{P} \cong M_{P} \otimes R \cong N_{Q} \otimes R \cong N\right)$. Here $\operatorname{Tr}$ denotes the normalized trace on $M_{n}(C)(\operatorname{Tr}(1)=1)$.

Let us now sketch the proof of the proposition. It is enough to show that $P_{p, q}$ and $Q_{p, q}$ can be chosen, satisfying (b) and such that $\Phi_{p, q}\left(P_{p, q}\right) \geq 1-9 \lambda_{q}^{\frac{1}{3}}$ and $\Psi_{p, q}\left(Q_{p, q}\right) \geq$ $1-9 \lambda_{q}^{\frac{1}{3}} ;$ if $\lambda_{q}^{\frac{1}{3}} \geq \frac{1}{9}$ then take $P_{p, q}$ and $Q_{p, q}$ to be any rank one projections. 
We can now drop the indices $(p, q)$. We keep the notation of lemma 2.2. Let $E=\left\{(k, j) ;\left|\log \left(\mu(k, j) / \mu^{\prime}(k, j)\right)\right| \leq 2 \lambda^{\frac{1}{3}}\right\}$. As

$$
\sum_{(k, j)}\left(1-\left(\frac{\mu(k, j)}{\mu^{\prime}(k, j)}\right)^{\frac{1}{2}}\right)^{2} \mu^{\prime}(k, j)=2\left(1-\rho\left(\mu, \mu^{\prime}\right)\right) \leq 4 \lambda
$$

and as for $(k, j) \notin E$,

$$
\left|\left(\frac{\mu(k, j)}{\mu^{\prime}(k, j)}\right)^{\frac{1}{2}}-1\right| \geq 1-\exp ^{-\lambda^{\frac{1}{3}}}
$$

we get that

$$
\mu^{\prime}(E) \geq 1-\frac{4 \lambda}{\left(1-\exp \left(-\lambda^{\frac{1}{3}}\right)\right)^{2}} \geq 1-5 \lambda^{\frac{1}{3}}
$$

In the same way

$$
\mu(E) \geq 1-\frac{4 \lambda}{\left(1-\exp \left(-\lambda^{\frac{1}{3}}\right)\right)^{2}} \geq 1-5 \lambda^{\frac{1}{3}}
$$

Put

$$
m_{k, j}=\frac{L !}{(L-|k|-j) ! k ! j !} \quad \text { and } \quad m_{k, j}^{\prime}=\frac{K !}{k !(K-|k|) !} \cdot \frac{J !}{j !(J-j) !}
$$

We have

$$
\frac{m_{k, j}}{m_{k, j}^{\prime}}=\frac{\mu(k, j)}{\mu^{\prime}(k, j)} \cdot \frac{\mu^{\prime}(0,0)}{\mu(0,0)}
$$

We distinguish two cases:

(1) $(0,0) \in E$. We have $\mid \log \left(m_{k, j} / m_{k, j}^{\prime} \mid \leq 4 \lambda^{\frac{1}{3}}\right.$, for $(k, j) \in E$. Let $q_{k, j}=$ $\min \left(m_{k, j}, m_{k, j}^{\prime}\right)$ and let $P_{k, j}$ (resp. $\left.Q_{k, j}\right)$ be a subprojection of dimension $q_{k, j}$ of the projection on the eigenspace of $\Phi\left(\right.$ resp. $\Psi$ ), relative to $\xi^{k} \lambda^{j} / \Lambda^{L}$ (resp. $\left.\xi^{k} \lambda^{j} /(\Lambda-\lambda)^{K}(1+\lambda)^{J}\right)$. Set $P=\sum_{(k, j) \in E} P_{k, j}$ and $Q=\sum_{(k, j) \in E} Q_{k, j}$. We have $\left(A_{P}, \Phi_{P}\right) \cong\left(B_{Q}, \Psi_{Q}\right)$ and

$$
\Phi(P) \geq \exp \left(-4 \lambda^{\frac{1}{3}}\right) \cdot \mu(E) \geq \exp \left(-4 \lambda^{\frac{1}{3}}\right)\left(1-5 \lambda^{\frac{1}{3}}\right) \geq 1-9 \lambda^{\frac{1}{3}} .
$$

Also, $\Psi(Q) \geq 1-9 \lambda^{\frac{1}{3}}$.

In this case, put $r=r^{\prime}=1$ and $(C, \chi)=\left(A_{P}, \Phi_{P}\right)$.

(2) $(0,0) \notin E$. Note that if $\lambda L / \Lambda<1$, then $K=L$ and

$$
\log \frac{\mu(0,0)}{\mu^{\prime}(0,0)}=\log \left(\frac{(1+\lambda)^{J}(\Lambda-\lambda)^{K}}{\Lambda^{L}}\right)=J \log (1+\lambda)+L \log \left(1-\frac{\lambda}{\Lambda}\right) .
$$

As $0 \leq \log (1+\lambda)-(\lambda /(1+\lambda)) \leq \lambda^{2} /(1+\lambda)$ and $-\lambda^{2} / \Lambda(\Lambda-\lambda) \leq \log (1-(\lambda / \Lambda))+$ $\lambda / \Lambda \leq 0$,

$$
\begin{aligned}
-\frac{\lambda}{\Lambda-\lambda} & \leq-\frac{L \lambda^{2}}{\Lambda(\Lambda-\lambda)} \leq \log \frac{\mu(0,0)}{\mu^{\prime}(0,0)}-\frac{J \lambda}{1+\lambda}+\frac{L \lambda}{\Lambda} \\
& \leq \frac{J \lambda^{2}}{1+\lambda} \leq \frac{L \lambda}{\Lambda} \lambda<\lambda .
\end{aligned}
$$

As $0 \leq(L \lambda / \Lambda)-(J \lambda /(1+\lambda))=(\lambda /(1+\lambda))[(L(1+\lambda) / \Lambda)-J] \leq \lambda /(1+\lambda)$, we get

$$
\left|\log \left(\frac{\mu(0,0)}{\mu^{\prime}(0,0)}\right)\right| \leq 2 \lambda \quad \text { and } \quad(0,0) \in E
$$


Hence, in our case, $\lambda L / \Lambda \geq 1$ and $J=[L(1+\lambda) / \Lambda] \geq L / \Lambda \geq \lambda^{-1}$. Moreover if $m_{k, j}^{\prime}=1$ and $(k, j) \neq(0,0)$, then either $K$ is equal to one of the $k_{i}$ 's or $J=j$. The $\mu$ and $\mu^{\prime}$-measure of these points is very small. Therefore, we will neglect them. (One can also show that these points do not belong to $E$ ).

Now, if $m_{k, j}^{\prime}>1$, then $m_{k, j}^{\prime} \geq J$. Let $r, r^{\prime} \in \mathbb{N}$ be such that $r^{\prime}$ is smaller than $J^{\frac{1}{2}}$ and

$$
\left|\frac{r}{r^{\prime}}-\frac{\mu^{\prime}(0,0)}{\mu(0,0)}\right| \leq J^{-\frac{1}{2}} \text {. }
$$

Let $q_{k, j}=\min \left(\left[m_{k, j} / r\right],\left[m_{k, j}^{\prime} / r^{\prime}\right]\right)$ and $P_{k, j}\left(\right.$ resp. $Q_{k, j}$ and $\left.R_{k, j}\right)$ be a subprojection of dimension $r q_{k, j}$ (resp. $r^{\prime} q_{k, j}$ and $q_{k, j}$ ) of the projection on the eigenspace of $\Phi\left(\right.$ resp. $\Psi$ ), relative to $\xi^{k} \lambda^{j} / \Lambda^{L}$ (resp. $\left.\xi^{k} \lambda^{j} /(\Lambda-\lambda)^{K}(1+\lambda)^{j}\right)$. Put $P=\sum_{(k, j) \in E} P_{k, j}$ $Q=\sum_{(k, j) \in E} Q_{k, j}, R=\sum_{(k, j) \in E} R_{k, j}$ and $(C, \chi)=\left(B_{R}, \Psi_{R}\right)$. We have

$$
\left(A_{P}, \Phi_{P}\right) \cong(C, \chi) \otimes\left(M_{r}(C), \operatorname{Tr}\right)
$$

and

$$
\left(B_{Q}, \Psi_{Q}\right) \cong(C, \chi) \otimes\left(M_{r^{\prime}}(C), \operatorname{Tr}\right) .
$$

It is now not difficult to estimate $\Phi(P)$ and $\Psi(Q)$.

\section{A sufficient condition for isomorphism of $\mathrm{ITPFI}_{2}$ factors}

The main result of this section is theorem 3.1, which gives a sufficient condition for two ITPFI $_{2}$ factors to be isomorphic. By theorem 2.1, any ITPFI factor $M$ of bounded type is isomorphic to some ITPFI ${ }_{2}$ factor $N$. In corollary 3.6, we give such an $N$ explicitly (in terms of the eigenvalues and multiplicities for $M$ ).

(3.1) THEOREM. Let $\left(\phi_{n}\right)_{n \geq 1}$ be a sequence of states on $M_{2}(C)$ with eigenvalues $\left\{1 /\left(1+\lambda_{n}\right), \lambda_{n} /\left(1+\lambda_{n}\right)\right\}, 0<\lambda_{n}<1$ and such that $\sum_{n \geq 1} \lambda_{n}<\infty$. Let $L_{n}$, $L_{n}^{\prime}$ be two sequences of integers. Let $M$ and $N$ be the ITPFI $_{2}$ factors:

$$
M=\bigotimes_{n \geq 1}\left(M_{2}(C), \phi_{n}\right)^{\otimes L_{n}} \text { and } N=\bigotimes_{n \geq 1}\left(M_{2}(C), \phi_{n}\right)^{\otimes L_{n}^{\prime}}
$$

Let $e_{n}=L_{n} \lambda_{n} /\left(1+\lambda_{n}\right), e_{n}^{\prime}=L_{n}^{\prime} \lambda_{n} /\left(1+\lambda_{n}\right)$, and $d_{n}$ be the closest integer to $e_{n}^{\prime}-e_{n}$; if $e_{n}^{\prime}-e_{n}=b+\frac{1}{2}, d_{n}=b$ or $d_{n}=b+1$ does not affect the convergence of the series (cf. remark 3.4). If

$$
\sum_{n \geq 1} \frac{\left(e_{n}^{\prime}-e_{n}-d_{n}\right)^{2}}{e_{n}+e_{n}^{\prime}}+\left(\frac{d_{n}}{e_{n}+e_{n}^{\prime}}\right)^{2}<\infty
$$

and $M$ is purely infinite, then $M$ and $N$ are isomorphic.

To simplify the coming notation, it will be convenient to assume that $L_{n}^{\prime} \geq L_{n}$, for all $n$. (If not, put $L_{n}^{\prime \prime}=\sup \left(L_{n}, L_{n}^{\prime}\right)$. Let $P$ be the corresponding $\operatorname{ITPFI}_{2}$ factor and compare $M$ and $N$ with $P$.)

We will prove that $M$ and $N$ have the same flow of weights. Let $M_{0}=$ $\otimes_{n \geq 1}\left(M_{2}(C), \phi_{n}\right)^{\otimes K_{n}}$. Let $\left(f_{n}\right)_{n \geq 1}$ be a sequence of integers and let $\mu_{n}$ be the probability measure on $\mathbb{Z}$, supported by $\left\{-f_{n}, 1-f_{n}, \ldots, K_{n}-f_{n}\right\}$ and given by

$$
\mu_{n}\left(k-f_{n}\right)=\frac{K_{n} !}{k !\left(K_{n}-k\right) !} \cdot \frac{\lambda^{k}}{(1+\lambda)^{K_{n}}} \quad \text { for } 0 \leq k \leq K_{n} \text {. }
$$


(The $f_{n}$ 's will be used in lemma 3.3). Let $(\Omega, \mu)$ be the product measure space $\prod_{n \geq 1}\left(\mathbb{Z}, \mu_{n}\right)$ and let $B \subset \Omega$ be a non-null set.

Let $\mathscr{R}$ be the equivalence relation on $B \times \mathbb{R}$ given by $(x, t) \mathscr{R}(y, s)$ iff there exists $q \in \mathbb{N}$ such that $x_{n}=y_{n}$, for all $n>q$, and

$$
-\sum_{n=1}^{q} x_{n} \log \lambda_{n}+t=-\sum_{n=1}^{q} y_{n} \log \lambda_{n}+s
$$

The flow of weights of $M_{0}$ is given by the action of $\mathbb{R}$ by translation on $B \times \mathbb{R} / \mathscr{R}$ (see appendix).

Using Kakutani's criterion and lemma 3.3 below, we are going to show that $M$ and $N$ have the same flow of weights. We first need the following:

(3.2) LemMA. Let $0<\lambda<1$ be a real number and $L \leq L^{\prime}$ be positive integers. Let $e=L \lambda /(1+\lambda), e^{\prime}=L^{\prime} \lambda /(1+\lambda), d$ be the closest integer to $e^{\prime}-e$ and $\delta=e^{\prime}-e-d$. Set

Then,

$$
V_{e}=\frac{\lambda^{d}}{(1+\lambda)^{L^{\prime}-L}} \cdot \prod_{i=1}^{d} \frac{1}{e+i} \prod_{i=1}^{L^{\prime}-L-d} \frac{1}{L-e+i} \prod_{i=1}^{L^{\prime}-L}(L+i) .
$$

One has

$$
V_{e} \geq\left(\frac{e}{(1+\lambda)(e+d)}\right)^{\frac{1}{2}} \exp \left(-\frac{(1+\lambda) \delta^{2}}{e^{\prime}}\right)
$$

Proof. For $t \geq 0$, put $g(t)=\log \left(L^{\prime}-d+t\right) /(e+t)$ and $h(t)=\log (L+t) /(L-e+t)$

$$
\begin{aligned}
\left(g(d)-g(0)+h\left(L^{\prime}-L-d\right)-h(0)\right) & =\log \left(\frac{L^{\prime}}{e+d} \cdot \frac{e}{L^{\prime}-d} \cdot \frac{L^{\prime}-d}{L^{\prime}-e-d} \cdot \frac{L-e}{L}\right) \\
& =\log \left(\frac{e}{e+d} \cdot \frac{L^{\prime}}{L^{\prime}-e-d} \cdot \frac{L-e}{L}\right) \\
\geq & \log \left(\frac{e}{e+d}\right)-\log (1+\lambda) .
\end{aligned}
$$

Now, the functions $g$ and $h$ are convex, so that $[g(i-1)+g(i)] / 2 \geq \int_{i-1}^{i} g(t) d t$. Therefore,

$$
\begin{aligned}
& \sum_{i=1}^{d} \frac{g(i-1)+g(i)}{2}+\sum_{i=1}^{L^{\prime}-L-d} \frac{h(i-1)+h(i)}{2} \\
& \geq \int_{0}^{d} g(t) d t+\int_{0}^{L^{\prime}-L^{-d}} h(t) d t \\
&= L^{\prime} \log L^{\prime}-(e+d) \log (e+d)-\left(L^{\prime}-d\right) \log \left(L^{\prime}-d\right)+e \log e \\
&+\left(L^{\prime}-d\right) \log \left(L^{\prime}-d\right)-\left(L^{\prime}-e-d\right) \log \left(L^{\prime}-e-d\right)-L \log L \\
&+(L-e) \log (L-e)
\end{aligned}
$$


Hence

$$
\begin{aligned}
V_{e} & \geq\left(\frac{e}{(1+\lambda)(e+d)}\right)^{\frac{1}{2}} \frac{L^{L^{\prime}} e^{e}(L-e)^{L-e}}{L^{L}(e+d)^{e+d}\left(L^{\prime}-e-d\right)^{L^{\prime}-e-d}} \frac{\lambda^{d}}{(1+\lambda)^{L^{\prime}-L}} \\
& =\left(\frac{e}{(1+\lambda)(e+d)}\right)^{\frac{1}{2}} \frac{\left(\frac{L^{\prime}}{1+\lambda}\right)^{L^{\prime}}\left(\frac{e}{\lambda}\right)^{e}(L-e)^{L-e}}{\left(\frac{L}{1+\lambda}\right)^{L}\left(\frac{e+d}{\lambda}\right)^{e+d}\left(L^{\prime}-e-d\right)^{L^{\prime}-e-d}} .
\end{aligned}
$$

Noting that $L /(1+\lambda)=e / \lambda=L-e$, we get

$$
\begin{aligned}
V_{e} & \geq\left(\frac{e}{(1+\lambda)(e+d)}\right)^{\frac{1}{2}} \frac{\left(\frac{L^{\prime}}{1+\lambda}\right)^{L^{\prime}}}{\left(\frac{e+d}{\lambda}\right)^{e+d}\left(L^{\prime}-e-d\right)^{L^{\prime}-e-d}} \\
& =\left(\frac{e}{(1+\lambda)(e+d)}\right)^{\frac{1}{2}}\left(\frac{\lambda L^{\prime}}{(1+\lambda)(e+d)}\right)^{e+d}\left(\frac{L^{\prime}}{(1+\lambda)\left(L^{\prime}-e-d\right)}\right)^{L^{\prime}-e-d} .
\end{aligned}
$$

But $\lambda L^{\prime} /(1+\lambda)=e^{\prime}, L^{\prime} /(1+\lambda)=L^{\prime}-e^{\prime}$. Using the inequality, $\log (a / b) \geq(a-b) / a$ we get:

$$
\begin{aligned}
(e+d) \log \frac{e^{\prime}}{e+d}+\left(L^{\prime}-e-d\right) \log \left(\frac{L^{\prime}-e^{\prime}}{L^{\prime}-e-d}\right) & \geq \frac{\delta(e+d)}{e^{\prime}}-\frac{\delta\left(L^{\prime}-e-d\right)}{L^{\prime}-e^{\prime}} \\
& =\frac{\delta\left(e^{\prime}-\delta\right)}{e^{\prime}}-\frac{\delta\left(L^{\prime}-e^{\prime}+\delta\right)}{L^{\prime}-e^{\prime}} \\
& =-\frac{\delta^{2}}{e^{\prime}}-\frac{\delta^{2}}{L^{\prime}-e^{\prime}}=-\frac{(1+\lambda) \delta^{2}}{e^{\prime}}
\end{aligned}
$$

Therefore,

$$
V_{e} \geq\left(\frac{e}{(1+\lambda)(e+d)}\right)^{\frac{1}{2}} \exp \left(-\frac{(1+\lambda) \delta^{2}}{e^{\prime}}\right)
$$

(3.3) LeMMA. Let $\mu$ and $\mu^{\prime}$ be the (probability) measures on $\mathbb{Z}$ given by

$$
\mu(k)=\frac{L !}{k !(L-k) !} \frac{\lambda^{k}}{(1+\lambda)^{L}}
$$

$(\mu(k)=0$ for $k<0$ or $k>L)$ and

$$
\mu^{\prime}(k)=\frac{L^{\prime} !}{(k+d) !\left(L^{\prime}-k-d\right) !} \frac{\lambda^{k+d}}{(1+\lambda)^{L^{\prime}}}
$$

$\left(\mu^{\prime}(k)=0\right.$ for $k<-d$ or $\left.k>L^{\prime}-d\right)$. Then we have

$$
\rho\left(\mu, \mu^{\prime}\right)=\sum_{k=0}^{L} \mu(k)^{\frac{1}{2}} \mu^{\prime}(k)^{\frac{1}{2}} \geq 1-\frac{\lambda}{2}-\frac{\delta^{2}}{e^{\prime}}-\frac{d^{2}}{2 e(e+d)}-\frac{d}{6 e^{2}} .
$$

Proof. For all $k, 0 \leq k \leq L$, we have

$$
\frac{\mu^{\prime}(k)}{\mu(k)}=V_{e} \prod_{i=1}^{d} \frac{e+i}{k+i} \prod_{i=1}^{L^{\prime}-L-d} \frac{L-e+i}{L-k+i}
$$


Hence,

$$
\log \frac{\mu^{\prime}(k)}{\mu(k)}=\log \left(V_{e}\right)-\sum_{i=1}^{d} \log \left(1+\frac{k-e}{e+i}\right)-\sum_{i=1}^{L^{\prime}-L-d} \log \left(1+\frac{e-k}{L-e+i}\right) .
$$

Using the inequalities $\log (1+x) \leq x-x^{2} / 2+x^{3} / 3$ and $\log (1+x) \leq x$ we get:

$$
\log \frac{\mu^{\prime}(k)}{\mu(k)} \geq \log V_{e}+\sum_{i=1}^{d}\left(\frac{e-k}{e+i}+\frac{(e-k)^{2}}{2(e+i)^{2}}+\frac{(e-k)^{3}}{3(e+i)^{3}}\right)+\sum_{i=1}^{L^{\prime}-L-d} \frac{k-e}{L-e+i}
$$

Now $\left(\mu^{\prime}(k) / \mu(k)\right)^{\frac{1}{2}} \geq 1+\frac{1}{2} \log \left(\mu^{\prime}(k) / \mu(k)\right)$. As $\sum_{k=0}^{L}(e-k) \mu(k)=0$,

$$
\sum_{k=0}^{L}(e-k)^{2} \mu(k)=\frac{L \lambda}{(1+\lambda)^{2}}=\frac{e}{1+\lambda}
$$

and

$$
\sum_{k=0}^{L}(e-k)^{3} \mu(k)=-\frac{L \lambda(1-\lambda)}{(1+\lambda)^{3}}=\frac{-e(1-\lambda)}{(1+\lambda)^{2}}
$$

(cf. [7]). We deduce

$$
\begin{aligned}
\rho\left(\mu, \mu^{\prime}\right) & \geq 1+\frac{1}{2} \log V_{e}+\frac{1}{2} \sum_{i=1}^{d} \frac{e}{2(1+\lambda)(e+i)^{2}}-\frac{e(1-\lambda)}{3(1+\lambda)^{2}(e+i)^{3}} \\
& \geq 1-\frac{1}{4} \log (1+\lambda)-\frac{1}{4} \log \frac{e+d}{e}-\frac{(1+\lambda) \delta^{2}}{2 e^{\prime}}+\frac{1}{4(1+\lambda)(e+d)^{2}}-\frac{e d}{6 e^{3}} \\
& \geq 1-\frac{\lambda}{4}-\frac{d}{4 e}-\frac{\delta^{2}}{e^{\prime}}+\frac{1}{4} \frac{d e}{(e+d)^{2}}-\frac{1}{4} \frac{\lambda d e}{(1+\lambda)(e+d)^{2}}-\frac{d}{6 e^{2}} \\
& \geq 1-\frac{\lambda}{4}-\frac{\delta^{2}}{e^{\prime}}-\frac{d\left((e+d)^{2}-e^{2}\right)}{4 e(e+d)^{2}}-\frac{\lambda}{4}-\frac{d}{6 e^{2}} \\
& \geq 1-\frac{\lambda}{2}-\frac{\delta^{2}}{e^{\prime}}-\frac{d^{2}(2 e+d)}{4 e(e+d)^{2}}-\frac{d}{6 e^{2}} \\
& \geq 1-\frac{\lambda}{2}-\frac{\delta^{2}}{e^{\prime}}-\frac{d^{2}}{2 e(e+d)}-\frac{d}{6 e^{2}}
\end{aligned}
$$

End of the proof of theorem 3.1. Let $L_{n}, L_{n}^{\prime}, \lambda_{n}, e_{n}, e_{n}^{\prime}, d_{n}$ be as in theorem 3.1 with $L_{n}^{\prime} \geq L_{n}$. Put $\delta_{n}=e_{n}^{\prime}-e_{n}-d_{n}$. Let $\mu_{n}$ and $\mu_{n}^{\prime}$ be the probability measures corresponding to $L_{n}, L_{n}^{\prime}, \lambda_{n}$ and $d_{n}$ as in lemma 3.3. Let $\nu$ and $\nu^{\prime}$ be the infinite product measures on $\prod_{n \geq 1} \mathbb{Z}: \nu=\bigotimes_{n \geq 1} \mu_{n}, \nu^{\prime}=\bigotimes_{n \geq 1} \mu_{n}^{\prime}$. For all $n, \mu_{n}$ is absolutely continuous with respect to $\mu_{n}^{\prime}$. Let $A=\left\{n \in \mathbb{N} \mid d_{n} \neq 0\right\}$. If $n \in A, e_{n}^{\prime}-e_{n} \leq \frac{3}{2} d_{n}$. Therefore

$$
\sum_{n \in A}\left(\frac{e_{n}^{\prime}-e_{n}}{e_{n}^{\prime}+e_{n}}\right)^{2}<\infty \text { and } \lim _{\substack{n \rightarrow \infty \\ n \in A}} \frac{e_{n}}{e_{n}^{\prime}}=1 \text {. }
$$

Changing finitely many $L_{n}$ 's, we may assume that for all $n \in A, e_{n} \geq e_{n}^{\prime} / 2$. By lemma 3.3 , we get

$$
\begin{aligned}
\sum_{n \geq 1}\left(1-\rho\left(\mu_{n}, \mu_{n}^{\prime}\right)\right) & \leq \sum_{n \geq 1} \frac{\lambda_{n}}{2}+\sum_{n \geq 1} \frac{\delta_{n}^{2}}{e_{n}^{\prime}}+\sum_{n \in A} \frac{d_{n}^{2}}{2 e_{n}\left(e_{n}+d_{n}\right)}+\sum_{n \in A} \frac{d_{n}}{6 e_{n}^{2}} \\
& \leq \sum_{n \geq 1} \frac{\lambda_{n}}{2}+\sum_{n \geq 1} \frac{\delta_{n}^{2}}{e_{n}^{\prime}}+\sum_{n \in A} \frac{2 d_{n}^{2}}{e_{n}^{\prime 2}}+\sum_{n \in A} \frac{2 d_{n}^{2}}{3 e_{n}^{\prime 2}}<+\infty
\end{aligned}
$$


By Kakutani's criterion $\left(\left[11\right.\right.$, p. 453]) $\nu$ is absolutely continuous with respect to $\nu^{\prime}$. By the above description of the flow of weights we deduce (using Krieger's theorem [12]) that $M$ and $N$ are isomorphic.

(3.4) Remark. Notice that the condition

$$
\sum_{n \geq 1} \frac{\left(e_{n}^{\prime}-e_{n}-d_{n}\right)^{2}}{e_{n}^{\prime}+e_{n}}+\frac{d_{n}^{2}}{\left(e_{n}^{\prime}+e_{n}\right)^{2}}<\infty
$$

can be replaced by the equivalent ones:

(i) There exists a sequence of integers $\left(d_{n}^{\prime}\right)_{n \geqslant 1}$ such that

$$
\sum_{n \geq 1} \frac{\left(e_{n}^{\prime}-e_{n}-d_{n}^{\prime}\right)^{2}}{e_{n}^{\prime}+e_{n}}+\frac{d_{n}^{\prime 2}}{\left(e_{n}^{\prime}+e_{n}\right)^{2}}<\infty .
$$

(ii)

$$
\sum_{n \geq 1} \frac{\left(e_{n}^{\prime}-e_{n}-d_{n}\right)^{2}}{e_{n}^{\prime}+e_{n}}+\frac{\left(e_{n}^{\prime}-e_{n}\right)^{2}}{\left(e_{n}+e_{n}^{\prime}+1\right)^{2}}<\infty .
$$

From theorem 3.1, we immediately get:

(3.5) Corollary. With the notation of theorem 3.1, if $\sum_{n \geq 1}\left|e_{n}^{\prime}-e_{n}\right| /\left(e_{n}+1\right)<\infty$, and $M$ is purely infinite, then $M$ and $N$ are isomorphic.

Proof. As $e_{n}^{\prime}-e_{n}-d_{n} \leq e_{n}^{\prime}+e_{n}$,

As $\left|e_{n}^{\prime}-e_{n}-d_{n}\right| \leq \frac{1}{2}$,

$$
\frac{\left(e_{n}^{\prime}-e_{n}-d_{n}\right)^{2}}{e_{n}^{\prime}+e_{n}} \leq\left|e_{n}^{\prime}-e_{n}-d_{n}\right| .
$$

$$
\begin{aligned}
\frac{\left(e_{n}^{\prime}-e_{n}-d_{n}\right)^{2}}{e_{n}^{\prime}+e_{n}} & \leq \inf \left\{\left|e_{n}^{\prime}-e_{n}-d_{n}\right|, \frac{\left|e_{n}^{\prime}-e_{n}-d_{n}\right|}{2\left(e_{n}^{\prime}+e_{n}\right)}\right\} \\
& \leq \frac{\left|e_{n}^{\prime}-e_{n}\right|}{\sup \left(1, e_{n}\right)} \leq \frac{2\left|e_{n}^{\prime}-e_{n}\right|}{e_{n}+1} .
\end{aligned}
$$

Moreover,

$$
\frac{\left(e_{n}^{\prime}-e_{n}\right)^{2}}{\left(e_{n}+e_{n}^{\prime}+1\right)^{2}} \leq \frac{\left|e_{n}^{\prime}-e_{n}\right|}{e_{n}+1} .
$$

We conclude the proof using remark 3.4 (ii).

Let $a$ be a positive real number and $\lambda_{q}$ be a sequence of real numbers with $e^{-(q+1) a}<$ $\lambda_{q} \leq e^{-q a}$. For an integer $k$, let $\psi_{k}$ be a state on $M_{2}(C)$ with eigenvalues $\left(1 /\left(1+\lambda_{k}\right)\right.$, $\left.\lambda_{k} /\left(1+\lambda_{k}\right)\right)$. Let $m \geq 1$ be an integer and, for each multi-index $p=\left(p_{1}, \ldots, p_{m}\right)$, $0 \leq p_{1} \leq p_{2} \leq \cdots \leq p_{m}$, let $\phi_{p}$ be a state with eigenvalues $\left(1 / \Lambda_{p}, \lambda_{p_{1}} / \Lambda_{p}, \cdots, \lambda_{p_{m}} / \Lambda_{p}\right)$ $\left(\Lambda_{p}=1+\sum_{i=1}^{m} \lambda_{p_{i}}\right)$.

(3.6) Corollary. Let $L_{p}$ be a sequence of integers indexed by the m-tuples $p=$ $\left(p_{1}, \ldots, p_{m}\right)$ with $0 \leq p_{1} \leq \cdots \leq p_{m}$. For $k \in \mathbb{N}$, let

$$
R_{k}=\left[\sum_{i=1}^{m} \sum_{\left\{p \mid p_{i}=k\right\}} \frac{L_{p}\left(1+\lambda_{k}\right)}{\Lambda_{p}}\right] .
$$

If $M=\bigotimes_{p}\left(M_{m+1}(C), \phi_{p}\right)^{\otimes L_{p}}$ is of type $\mathrm{III}_{0}$, then it is isomorphic to $N=$ $\otimes_{k}\left(M_{2}(C), \psi_{k}\right)^{\otimes R_{k}}$. 
Proof. We prove corollary 3.6 by induction on $m$. If $m=1$ there is nothing to prove.

Let $M=\otimes_{(p, q)}\left(M_{m+2}(C), \phi_{p, q}\right)^{\otimes L_{p, q}}$ be an ITPFI $_{m+2}$ factor written as in the proof of theorem 2.1. Let

$$
M_{1}=\bigotimes_{p, q}\left[\left(M_{m+1}(C), \phi_{p}\right)^{\otimes K_{p, q} \otimes}\left(M_{2}(C), \phi_{q}\right)^{\otimes J_{p, q}}\right]
$$

where $\quad K_{p, q}=L_{p, q}-\left[\lambda_{q} L_{p, q} / \Lambda_{p, q}\right], \quad\left(\Lambda_{p, q}=1+\sum_{i=1}^{m} \lambda_{p_{i}}+\lambda_{q}=\Lambda_{p}+\lambda_{q}\right), \quad J_{p, q}=$ $\left[\left(\left(1+\lambda_{q}\right) / \Lambda_{p, q}\right) L_{p, q}\right]$.

By the proof of theorem 2.1, M and $M_{1}$ are isomorphic. By induction, $M_{1}$ is isomorphic to $N_{1}=\bigotimes_{k}\left(M_{2}(C), \phi_{k}\right)^{\otimes S_{k}}$ where

$$
S_{k}=\left[\sum_{i=1}^{m} \sum_{\substack{p, q \\ p_{i}=k}} \frac{K_{p, q}\left(1+\lambda_{k}\right)}{\Lambda_{p}}\right]+\sum_{p} J_{p, k}
$$

As $K_{p, q} / \Lambda_{p} \geq L_{p, q} / \Lambda_{p, q}$, we have:

as

$$
\begin{aligned}
S_{k} & \geq\left[\sum_{i=1}^{m} \sum_{\left\{(p, q) \mid p_{i}=k\right\}} \frac{L_{p, q}\left(1+\lambda_{k}\right)}{\Lambda_{p, q}}\right]+\sum_{p}\left[\frac{L_{p, k}\left(1+\lambda_{k}\right)}{\Lambda_{p, k}}\right] \\
& \geq R_{k}-\frac{(k+m) !}{k ! m !}
\end{aligned}
$$

$$
R_{k}=\left[\sum_{i=1}^{m} \sum_{\left\{(p, q) \mid p_{i}=k\right\}} \frac{L_{p, q}\left(1+\lambda_{k}\right)}{\Lambda_{p, q}}+\sum_{p} \frac{L_{p, k}\left(1+\lambda_{k}\right)}{\Lambda_{p, k}}\right] .
$$

On the other hand,

$$
S_{k} \leq\left[\sum_{i=1}^{m} \sum_{\left\{(p, k) \mid p_{i}=k\right\}} \frac{L_{p, q}\left(1+\lambda_{k}\right)}{\Lambda_{p}}+\sum_{p} \frac{L_{p, k}\left(1+\lambda_{k}\right)}{\Lambda_{p, k}}\right] .
$$

Note that for $k \leq q$ we have

Hence

$$
\frac{\Lambda_{p, q}}{\Lambda_{p, q}-\lambda_{q}} \leq \frac{\Lambda_{p, q}-\lambda_{q}+\lambda_{k}}{\Lambda_{p, q}-\lambda_{q}} \leq 1+\lambda_{k}
$$

$$
S_{k}<\left(1+\lambda_{k}\right)\left(R_{k}+1\right) .
$$

We have $\lambda_{k}\left(R_{k}-S_{k}\right) \leq \lambda_{k}(k+m) ! / k ! m ! \quad$ and $\quad\left(S_{k}-\left(R_{k}+1\right)\right) /\left(R_{k}+1\right)<\lambda_{k}$. Therefore,

$$
\left|\frac{\lambda_{k}\left(R_{k}-S_{k}\right)}{\lambda_{k} R_{k}+1}\right| \leq \lambda_{k} \frac{(k+m) !}{k ! m !}
$$

so that

$$
\sum_{k=1}^{+\infty}\left|\frac{\lambda_{k}\left(R_{k}-S_{k}\right)}{\lambda_{k} R_{k}+1}\right|<+\infty .
$$

By corollary 3.5, $N=\bigotimes_{k=1}^{\infty}\left(M_{2}(C), \psi_{k}\right)^{\otimes R_{k}}$ is isomorphic to $N_{1}$.

Theorem 3.1 gives also a (negative) answer to problem 7 of [16, p. 37].

(3.7) CoRollary. Let $L_{k}$ be a sequence of integers and $\lambda, 0<\lambda<1$, a real number. Let $\phi_{k}$ be a state on $M_{2}(C)$ with eigenvalues $\left\{1 /\left(1+\lambda^{k}\right), \lambda^{k} /\left(1+\lambda^{k}\right)\right\}$. Let $M$ be the ITPFI $I_{2}$ factor: $M=\bigotimes_{k \geq 1}\left(M_{2}(C), \phi_{k}\right)^{\otimes L_{k}}$. 
Then if $M$ is not of type $I$, there exists a sequence $L_{k}^{\prime}$ such that $\sum_{k \geq 1} \lambda^{k}\left|L_{k}^{\prime}-L_{k}\right|=+\infty$ and $M$ is isomorphic to the $\mathrm{ITPFI}_{2}$ factor $N=\bigotimes_{k \geq 1}\left(M_{2}(C), \phi_{k}\right)^{\otimes L_{k}^{\prime}}$.

Proof. The factor $M$ not being of type $I$, the sequence $\left(L_{k} \lambda^{k}\right)$ is not summable. Therefore there exists a sequence $\left(a_{k}\right) \in \ell_{2}(\mathbb{N})$ such that $\sum_{k \geq 1}\left(L_{k} \lambda^{k}\right)^{\frac{1}{2}} a_{k}=+\infty$. Let $L_{k}^{\prime}=L_{k}+\left[a_{k}\left(L_{k} \lambda^{-k}\right)^{\frac{1}{2}}\right]$ We have $\sum_{k}\left(L_{k}^{\prime}-L_{k}\right) \lambda^{k}=+\infty$ and $\sum_{k} \lambda^{k}\left(L_{k}^{\prime}-L_{k}\right)^{2} / L_{k}<+\infty$. This implies, by remark 3.4(i), that $M$ and $N$ are isomorphic.

\section{An example}

Theorem 3.1 does not admit a converse in general (for example, the Powers factors of type III $_{\lambda}$ or the Araki \& Woods type III $_{1}$ factor can be written in many completely different ways).

However in some situations it does admit a converse (proposition 4.4). This yields a quite surprising example (4.5).

Let $L_{n}$ be a sequence of positive integers and let $\lambda_{n}$ be a sequence of positive real numbers. Let $\mu_{n}$ be the measure on $\mathbb{Z}$ (supported by $\left\{0, \ldots, L_{n}\right\}$ ) corresponding to $\left(L_{n}, \lambda_{n}\right)$ and let $\mu=\bigotimes_{n \geq 1} \mu_{n}$ be the product measure on $\prod_{n \geq 1} \mathbb{Z}$.

(4.1) Definition. The sequence $\left(L_{n}, \lambda_{n}\right)$ is said to satisfy condition $\mathrm{C}$ if there exists $\varepsilon>0$ and sequences $a_{n}, b_{n}$ of integers such that

(i) $\mu\left(\left\{x=\left(x_{n}\right)_{n \geq 1} \in \prod_{n \geq 1} \mathbb{Z} \mid a_{n} \leq x_{n} \leq b_{n}\right.\right.$, for all $\left.\left.n \geq 1\right\}\right)>0$;

(ii) for all $n \geq 1,-\log \lambda_{n} \geq \varepsilon-\sum_{k=1}^{n-1} 2\left(b_{k}-a_{k}\right) \log \lambda_{k}$.

A much stronger condition was used by Araki \& Woods to prove the existence of type $\mathrm{III}_{0}$ factors $([1, \S 10]$, cf. also [15]).

(4.2) Remark. Let $a_{n}, b_{n}$ be as in definition 4.1 and let $B=\left\{x \in \prod_{n \geq 1} \mathbb{Z} \mid a_{n} \leq x_{n} \leq b_{n}\right\}$. Using the description of the flow of weights given in the appendix, $B \times \mathbb{R}$ maps onto the flow space. Condition (ii) says that $B \times[0, \varepsilon)$ maps injectively to the flow space. In particular if $\sum_{n \geq 1} L_{n} \lambda_{n}=+\infty$ the corresponding factor is of type III $_{0}$.

(4.3) Lemma. Let $\left(L_{n}, \lambda_{n}\right),\left(L_{n}^{\prime}, \lambda_{n}\right)$ satisfy condition C. Assume that the corresponding factors are isomorphic.

Then, there exists a sequence of integers $\left(c_{n}\right)_{n \geq 1}$ such that $\phi_{*} \mu$ and $\mu^{\prime}$ are not (mutually) singular, where $\phi: \prod_{n \geq 1} \mathbb{Z} \rightarrow \prod_{n \geq 1} \mathbb{Z}$ is defined by $\phi(x)_{n}=x_{n}+c_{n}$. $(\mu$ and $\mu^{\prime}$ denote the measures on $\prod_{n \geq 1} \mathbb{Z}$ associated with $\left(L_{n}, \lambda_{n}\right)$ and $\left(L_{n}^{\prime}, \lambda_{n}\right)$, as above.) Proof. Let $a_{n}, b_{n}, a_{n}^{\prime}, b_{n}^{\prime}, \eta\left(=\inf \left(\varepsilon, \varepsilon^{\prime}\right)\right)$ be given by definition 4.1.

Let $B_{0}=\prod_{n \geq 1}\left\{a_{n}, \ldots, b_{n}\right\}, B_{0}^{\prime}=\prod_{n \geq 1}\left\{a_{n}^{\prime}, \ldots, b_{n}^{\prime}\right\}$. Let $T_{0}: B_{0} \rightarrow B_{0}, T_{0}^{\prime}: B_{0}^{\prime} \rightarrow B_{0}^{\prime}$ be the odometers. Let $x \in B_{0}$ and $p \in \mathbb{N}$. Then $\left(T_{0}^{p} x\right)_{n}=x_{n}$ for $n$ large enough. Let

$$
\xi(x, p)=-\sum_{n \geq 1}\left(\left(T_{0}^{p} x\right)_{n}-x_{n}\right) \log \lambda_{n}
$$

and

$$
\xi^{\prime}\left(x^{\prime}, p\right)=-\sum_{n \geq 1}\left(\left(T_{0}^{\prime p} x^{\prime}\right)_{n}-x_{n}^{\prime}\right) \log \lambda_{n} \quad\left(x^{\prime} \in B_{0}^{\prime}\right) .
$$

As the corresponding factors are isomorphic, there exists an isomorphism between their flows of weights. 
These flows are constructed over the base transformations $\left(B_{0}, \mu, T_{0}\right),\left(B_{0}^{\prime}, \mu^{\prime}, T_{0}^{\prime}\right)$ with the ceiling functions $\xi(x, 1)$ and $\xi^{\prime}\left(x^{\prime}, 1\right)$. The isomorphism of the flows means that there exist: non-null subsets $B_{1}, B_{1}^{\prime}$ of $B_{0}$ and $B_{0}^{\prime}$; a measure-class preserving isomorphism $\phi: B_{1} \rightarrow B_{1}^{\prime}$ intertwining the induced transformations $T_{1}$ and $T_{1}^{\prime}$; a measurable map $\alpha: B_{1} \rightarrow \mathbb{R}$ such that if $\xi_{1}$ and $\xi_{1}^{\prime}$ are the induced ceiling functions $\left(\xi_{1}(x)=\xi(x, m(x))\right.$ if $\left.T_{1}(x)=T_{0}^{m(x)}(x)\right)$,

$$
\xi_{1}^{\prime}(\phi(x))=\xi_{1}(x)+\alpha\left(T_{1}(x)\right)-\alpha(x)
$$

for almost all $x$.

There exists an interval $I$ in $\mathbb{R}$ of length smaller than $\eta$ such that $\mu\left(\alpha^{-1}(I)\right) \neq 0$. Let $B=\alpha^{-1}(I), B^{\prime}=\phi(B)$. Let $T$ and $T^{\prime}$ be the induced transformations in $B$ and $B^{\prime}$.

For $x \in B$ (resp. $x^{\prime} \in B^{\prime}$ ), let $p(x)$ (resp. $p^{\prime}\left(x^{\prime}\right)$ ) be the integer satisfying $T(x)=$ $T_{0}^{p(x)}(x)\left(\right.$ resp. $\left.\left.T^{\prime}\left(x^{\prime}\right)\right)=T_{0}^{\prime p^{\prime}\left(x^{\prime}\right)}\left(x^{\prime}\right)\right)$. Let $q(x)$ be defined by $T(x)=T_{1}^{q(x)}(x)$. Note that $T^{\prime}(\phi(x))=\phi(T(x))=T_{1}^{\prime q(x)}(\phi(x))$.

Put $\xi(x)=\xi(x, p(x))=\sum_{i=0}^{q(x)-1} \xi_{1}\left(T_{1}^{i}(x)\right) ; \xi^{\prime}\left(x^{\prime}\right)=\xi^{\prime}\left(x^{\prime}, p^{\prime}\left(x^{\prime}\right)\right)$. Note that we still have $\xi^{\prime}(\phi(x))=\xi(x)+\alpha(T x)-\alpha(x)$. (As $\xi^{\prime}(\phi(x))-\xi(x)=\sum_{i=0}^{q(x)-1}\left(\xi_{1}\left(\phi\left(T_{1}^{i}(x)\right)\right)-\right.$ $\left.\xi_{1}\left(T_{1}^{i}(x)\right)\right)=\alpha(T x)-\alpha(x)$.)

Now, for all $x$ and $p, x^{\prime}$ and $p^{\prime}$, we have

$$
\left|\xi(x, p)-\xi^{\prime}\left(x^{\prime}, p^{\prime}\right)\right| \leq \eta \Rightarrow \xi(x, p)=\xi^{\prime}\left(x^{\prime}, p^{\prime}\right)
$$

(condition $\mathrm{C}$ ). We derive $\left.\xi^{\prime}(\phi x)\right)=\xi(x)$. Hence,

$$
-\sum_{n \geq 1}\left((T x)_{n}-x_{n}\right) \log \lambda_{n}=-\sum_{n \geq 1}\left(\left(T^{\prime} \phi(x)\right)_{n}-(\phi(x))_{n}\right) \log \lambda_{n}
$$

By condition C, we get $(T x)_{n}-x_{n}=\left(T^{\prime} \phi(x)\right)_{n}-(\phi(x))_{n}$ for all $n$, which means $(\phi(x))_{n}-x_{n}=(\phi T(x))_{n}-(T x)_{n}$. By ergodicity of $T$, we get that $(\phi(x))_{n}-x_{n}$ is essentially constant (equal to an integer $c_{n}$ ) for all $n$. Moreover, $\left.\phi_{*} \mu\right|_{B}$ and $\left.\mu^{\prime}\right|_{B^{\prime}}$ are equivalent. Therefore, if we define $\phi: \prod_{n \geq 1} \mathbb{Z} \rightarrow \prod_{n \geq 1} \mathbb{Z}$, by $(\phi(x))_{n}=x_{n}+c_{n}$, we get that $\phi_{*} \mu$ and $\mu^{\prime}$ are not singular.

The following proposition is a partial converse of theorem 3.1.

(4.4) Proposition. Let $\left(L_{n}, \lambda_{n}\right),\left(L_{n}^{\prime}, \lambda_{n}\right)$ satisfy condition C. If the corresponding $\mathrm{ITPFI}_{2}$ factors are isomorphic, then

$$
\sum_{n \geq 1} \frac{\left(e_{n}^{\prime}-e_{n}-d_{n}\right)^{2}}{e_{n}^{\prime}+e_{n}}+\left(\frac{d_{n}}{e_{n}^{\prime}+e_{n}}\right)^{2}<\infty,
$$

where $e_{n}=L_{n} \lambda_{n} /\left(1+\lambda_{n}\right), e_{n}^{\prime}=L_{n}^{\prime} \lambda_{n} /\left(1+\lambda_{n}\right)$ and $d_{n}$ is the closest integer to $e_{n}^{\prime}-e_{n}$.

Proof. By lemma 4.3 , there exists a sequence $\left(c_{n}\right)_{n \geq 1}$ of integers such that $\phi_{*} \mu$ and $\mu^{\prime}$ are not singular.

Let $\left(a_{n}\right)_{n \geq 1}$ be a sequence of real numbers such that $\sum_{n \geq 1} a_{n}^{2}\left(e_{n}+e_{n}^{\prime}\right) /\left(1+\lambda_{n}\right)<\infty$ and $a_{n}\left(e_{n}^{\prime}-e_{n}-c_{n}\right) \geq 0$. As we have $\sigma_{\mu}^{2}\left(x_{n}\right)=e_{n} /\left(1+\lambda_{n}\right)$ and $\sigma_{\mu}^{2},\left(x_{n}\right)=e_{n}^{\prime} /\left(1+\lambda_{n}\right)$ and using a theorem of Kolmogorov (cf. theorem $B$ of $\$ 46$ of [9]), we get: $\sum_{n \geq 1} a_{n}\left(x_{n}-e_{n}\right)$ converges $\mu$-a.e., $\sum_{n \geq 1} a_{n}\left(x_{n}-c_{n}-e_{n}\right)$ converges $\phi_{*} \mu$-a.e., also $\sum_{n \geq 1} a_{n}\left(x_{n}-e_{n}^{\prime}\right)$ converges $\mu^{\prime}$-a.e. 
As $\phi_{*} \mu$ and $\mu^{\prime}$ are not singular, we get

$$
\sum_{n \geq 1} a_{n}\left(e_{n}^{\prime}-c_{n}-e_{n}\right)<\infty .
$$

If $\left(e_{n}^{\prime}-e_{n}-c_{n}\right) /\left(e_{n}^{\prime}+e_{n}\right)^{\frac{1}{2}} \notin \ell_{2}(\mathbb{N})$, there exists a sequence $\left(b_{n}\right)_{n \geq 1} \in \ell_{2}(\mathbb{N})$ such that $\sum_{n \geq 1}\left|b_{n}\left(e_{n}^{\prime}-e_{n}-c_{n}\right)\right| /\left(e_{n}^{\prime}+e_{n}\right)^{\frac{1}{2}}=\infty$. Setting $\left|a_{n}\right|=\left|b_{n}\right| /\left(e_{n}^{\prime}+e_{n}\right)^{\frac{1}{2}}$, we get a contradiction. Therefore, $\left(e_{n}^{\prime}-e_{n}-c_{n}\right) /\left(e_{n}^{\prime}+e_{n}\right)^{\frac{1}{2}} \in \ell_{2}(\mathbb{N})$ and $\sum_{n \geq 1}\left(e_{n}^{\prime}-e_{n}-d_{n}\right)^{2} /$ $\left(e_{n}^{\prime}+e_{n}\right)<\infty$, where $d_{n}$ denotes the closest integer to $e_{n}^{\prime}-e_{n}$.

Put $\delta_{n}=e_{n}^{\prime}-e_{n}-c_{n}$, for all $n \geq 1$. We have:

$$
\begin{aligned}
\sigma_{\mu}^{2}\left(\left(x_{n}-e_{n}-\frac{\delta_{n}}{2}\right)^{2}\right)= & \frac{L_{n} \lambda_{n}}{\left(1+\lambda_{n}\right)^{3}}\left(\frac{2 L_{n} \lambda_{n}}{1+\lambda_{n}}+\frac{1-4 \lambda_{n}+\lambda_{n}^{2}}{1+\lambda_{n}}\right) \\
& -2 \delta_{n} \frac{L_{n} \lambda_{n}\left(1-\lambda_{n}\right)}{\left(1+\lambda_{n}\right)^{3}}+\frac{\delta_{n}^{2} L_{n} \lambda_{n}}{\left(1+\lambda_{n}\right)^{2}} \\
\leq & 2 e_{n}^{2}+e_{n}+2\left|\delta_{n}\right| e_{n}+\delta_{n}^{2} e_{n} .
\end{aligned}
$$

Also,

$$
\sigma_{\mu}^{2} \cdot\left(\left(x_{n}-e_{n}^{\prime}+\frac{\delta_{n}}{2}\right)^{2}\right) \leq 2 e_{n}^{\prime 2}+e_{n}^{\prime}+2\left|\delta_{n}\right| e_{n}^{\prime}+\delta_{n}^{2} e_{n}^{\prime} .
$$

As $\sum_{n \geq 1} \delta_{n}^{2} /\left(e_{n}+e_{n}^{\prime}\right)<+\infty$, we may assume $\left|\delta_{n}\right| \leq\left(e_{n}+e_{n}^{\prime}\right)^{\frac{1}{2}}$ for all $n$. We get

$$
\begin{aligned}
& \sigma_{\mu}^{2}\left(\left(x_{n}-e_{n}-\frac{\delta_{n}}{2}\right)^{2}\right)+\sigma_{\mu}^{2} \cdot\left(\left(x_{n}-e_{n}^{\prime}+\frac{\delta_{n}}{2}\right)^{2}\right) \\
& \quad \leq 2\left(e_{n}+e_{n}^{\prime}\right)^{2}+e_{n}+e_{n}^{\prime}+2\left(e_{n}+e_{n}^{\prime}\right)^{\frac{3}{2}}+\left(e_{n}+e_{n}^{\prime}\right)^{2} \\
& \quad \leq 4\left(e_{n}+e_{n}^{\prime}\right)^{2}+2\left(e_{n}+e_{n}^{\prime}\right) \\
& \quad \leq 4\left(e_{n}+e_{n}^{\prime}+1\right)^{2} .
\end{aligned}
$$

If $\sum_{n \geq 1}\left(e_{n}-e_{n}^{\prime}\right)^{2} /\left(e_{n}+e_{n}^{\prime}+1\right)^{2}=\infty$, there exists a sequence $\left(b_{n}\right)_{n \geq 1}$ such that $\sum_{n \geq 1} b_{n}^{2}<\infty, \quad b_{n}\left(e_{n}-e_{n}^{\prime}\right) \geq 0$ and $\sum_{n \geq 1} b_{n}\left(e_{n}-e_{n}^{\prime}\right) /\left(e_{n}+e_{n}^{\prime}+1\right)=\infty$. Put $a_{n}=$ $b_{n} /\left(e_{n}+e_{n}^{\prime}+1\right)$.

As $\sum_{n \geq 1} a_{n}^{2}\left(\sigma_{\mu}^{2}\left(\left(x_{n}-e_{n}-\left[\delta_{n} / 2\right]\right)^{2}\right)+\sigma_{\mu}^{2} \cdot\left(\left(x_{n}-e_{n}^{\prime}+\left[\delta_{n} / 2\right]\right)^{2}\right)\right)<\infty$, by a theorem of Kolmogorov (cf. [9, $\$ 46$, theorem B]), we get

$$
\sum_{n \geq 1} a_{n}\left(\left(x_{n}-e_{n}-\left(\delta_{n} / 2\right)\right)^{2}-e_{n} /\left(1+\lambda_{n}\right)-\delta_{n}^{2} / 4\right)
$$

converges $\mu$-a.e. Hence,

$$
\sum_{n \geq 1} a_{n}\left(\left(x_{n}-e_{n}^{\prime}+\left(\delta_{n} / 2\right)\right)^{2}-e_{n} /\left(1+\lambda_{n}\right)-\delta_{n}^{2} / 4\right)
$$

converges $\phi_{*} \mu$-a.e. Also,

$$
\left.\sum_{n \geq 1} a_{n}\left(\left(x_{n}-e_{n}^{\prime}+\left(\delta_{n} / 2\right)\right)^{2}-e_{n}^{\prime} /\left(1+\lambda_{n}\right)-\delta_{n}^{2} / 4\right)\right)
$$

converges $\mu^{\prime}$-a.e. As $\phi_{*} \mu$ and $\mu^{\prime}$ are not singular, we get

$$
\sum_{n \geq 1} a_{n}\left(e_{n}-e_{n}^{\prime}\right) /\left(1+\lambda_{n}\right)<\infty,
$$

which contradicts our assumption $\sum_{n \geq 1} b_{n}\left(e_{n}-e_{n}^{\prime}\right) /\left(e_{n}+e_{n}^{\prime}+1\right)=\infty$.

The proposition now follows from remark 3.4. 
(4.5) Example. Let $\lambda \in(0,1)$. Put $\lambda_{k}=\lambda^{(2 k) !}(k \geq 1)$. Let

$$
L_{k}=\left[\left(\frac{1+\lambda_{k}}{\lambda_{k}}\right) k\right], \quad L_{k}^{\prime}=\left[\left(\frac{1+\lambda_{k}}{\lambda_{k}}\right)\left(k+\frac{1}{2}\right)\right], \quad L_{k}^{\prime \prime}=\left[\left(\frac{1+\lambda_{k}}{\lambda_{k}}\right)(k+1)\right] .
$$

One easily checks that the sequences $\left(L_{k}, \lambda_{k}\right),\left(L_{k}^{\prime}, \lambda_{k}\right),\left(L_{k}^{\prime \prime}, \lambda_{k}\right)$ satisfy condition C (taking $a_{k}=0, b_{k}=k(k+1)$ and using Tchebyshev's inequality).

Let $M, N, P$ be the corresponding factors. Using theorem 3.1, we get that $M$ and $P$ are isomorphic; using proposition 4.4, we get that $M$ and $N$ are not isomorphic. Note that, for all $k, L_{k} \leq L_{k}^{\prime} \leq L_{k}^{\prime \prime}$.

Using theorem 3.1, again we get that $M \otimes M$ and $N \otimes N$ are isomorphic.

Let $\Lambda_{k}=\left[\left(1+\lambda_{k}\right) / 2 \lambda_{k}\right]$ and let $Q$ be the factor corresponding to $\left(\Lambda_{k}, \lambda_{k}\right)$. We have $M \otimes Q(\cong N)$ is not isomorphic to $M$, though $M \otimes Q \otimes Q(\cong P)$ is isomorphic to $M$.

Taking $\Lambda_{k}=\left[\left(1+\lambda_{k}\right) / q \lambda_{k}\right]$, where $q$ is an integer, we get factors $M$ and $Q$ such that $M \otimes Q^{\otimes j}, j=0, \ldots, q-1$, are pairwise non-isomorphic but $M \otimes Q^{\otimes q}$ is isomorphic to $M$.

\section{Appendix}

In $\S \S 2,3$ we use only a partial determination of the Connes-Takesaki flow of weights. In order to make the exposition reasonably self-contained, we outline the relevant aspects of this construction. In part (A) we set it in the general framework of equivalence relations. In part (B) we specialize it to the ITPFI case. Finally in parts (C) and (D) we indicate how this partial construction is used in $\S \S 3(\mathrm{C})$ and 2(D).

(A) Let $\mathscr{R}_{0}$ be a type III discrete ergodic equivalence relation on the Lebesgue measure space $\left(\Omega_{0}, P\right)$. Let $M=M\left(\mathscr{R}_{0}\right)$ be the corresponding factor.

By [5, theorem II 6.2] (cf. also [4], [8], [10], [13], [14]) the flow of weights of $M$ is given by the action of $\mathbb{R}$ by translation in $\Omega_{0} \times \mathbb{R} / \tilde{\mathscr{R}}_{0}$ (this quotient stands for the ergodic decomposition) where $\tilde{\mathscr{R}}_{0}$ is the equivalence relation in $\left(\Omega_{0} \times \mathbb{R}, P \times m\right)$ given by $(x, t) \tilde{R}_{0}(y, s)$ iff $x \mathscr{R}_{0} y$ and $s=t-\log \delta(x, y)$ where $\delta(x, y)$ is the module of $P$ (cf. [4], [6, p. 434]) ( $m$ is a finite measure on $\mathbb{R}$ equivalent to the Lebesgue measure). Note that as $\left(\Omega_{0}, P, \mathscr{R}_{0}\right)$ is weakly equivalent to its restriction $\left(A, P, \mathscr{R}_{0 \mid A}\right)$ for $A \subseteq \Omega_{0}, P(A) \neq 0$, the flow of weights may also be realized in $A \times \mathbb{R} / \tilde{\mathscr{R}}_{0 \mid A \times \mathbb{R}}$.

This ergodic decomposition has explicitly been determined in some cases (cf. [3], $[10, \S I .6]$, it can also be obtained in $\S 4$ of this paper). In general, however, it seems to be a problem.

Let $\tilde{\mathscr{R}}_{1}$ be another equivalence relation, $\tilde{\mathscr{R}}_{1} \subseteq \tilde{\mathscr{R}}_{0}$. The ergodic decomposition $\left(\Omega_{0} \times \mathbb{R}\right) / \tilde{R}_{0}$ can be obtained in two steps, computing first $(Y, \mu)=\left(\Omega_{0} \times \mathbb{R}, P \times\right.$ $m) / \tilde{\mathscr{R}}_{1}$ and then $(Y, \mu) / \tilde{\mathscr{R}}$ where $\tilde{\mathscr{R}}$ is a discrete equivalence relation given below. The interest of this construction is that, in the cases we are interested in, we are able to compute both the quotient $(Y, \mu)$ and the equivalence relation $\tilde{R}$.

The measure $P \times m$ admits the disintegration $P \times m=\int_{Y} v_{y} d \mu(y)$ where the $v_{y}$ are pairwise singular $\tilde{\mathscr{R}}_{1}$-quasi-invariant and ergodic measures on $\Omega \times \mathbb{R}$. Then $\tilde{\mathscr{R}}$ is defined by any of the equivalent conditions: 
$y \tilde{\mathscr{R}} y^{\prime}$ iff (i) for all measurable subsets $E, E^{\prime}$ of $\Omega_{0} \times \mathbb{R}$ such that $v_{y}(E) \neq 0, v_{y^{\prime}}\left(E^{\prime}\right) \neq 0$ there exist $x \in E, x^{\prime} \in E^{\prime}$ with $x \tilde{\mathscr{R}}_{0} x^{\prime}$, or

(ii) there exists a partial borel transformation $\phi$ whose graph is contained in the graph of $\tilde{\mathscr{R}}_{0}$ such that $v_{y}(\operatorname{dom} \phi)=1$ and that $\phi_{*}\left(v_{y}\right)$ and $v_{y^{\prime}}$ are not singular.

If $\tilde{\mathscr{R}}_{0}$ is defined by the countable set $\left(g_{n}\right)_{n \geq 1}$ of transformations of $\Omega_{0} \times \mathbb{R}$, we also have the condition

(iii) $m_{y}=\sum_{n \geq 1} 2^{-n} g_{n^{*}}\left(v_{y}\right)$ and $m_{y^{\prime}}=\sum_{n \geq 1} 2^{-n} g_{n^{*}}\left(v_{y^{\prime}}\right)$ are equivalent.

(To see the equivalence of (i), (ii) and (iii) note that $m_{y}$ and $m_{y^{\prime}}$ are $\tilde{\mathscr{R}}_{0}$-quasiinvariant and ergodic, hence either singular or equivalent).

(B) In the ITPFI case, $\left(\Omega_{0}, P\right)=\prod_{n \geq 1}\left(X_{n}, P_{n}\right)$ where $\left(X_{n}, P_{n}\right)$ is a finite probability space for all $n$. The equivalence relation $\mathscr{R}_{0}$ is given by $\omega \mathscr{R}_{0} \omega^{\prime}$ iff $\omega_{n}=\omega_{n}^{\prime}$ for all but finitely many $n$ 's. There may be some multiplicity in the eigenvalues, which means, in our setting, that there may be $x, x^{\prime} \in X_{n}$ with $P_{n}(x)=P_{n}\left(x^{\prime}\right)\left(x \neq x^{\prime}\right)$. Let $\mathscr{S}_{n}$ be an equivalence relation in $X_{n}$ preserving $P_{n}\left(\right.$ i.e. if $\left.x \mathscr{S}_{n} x^{\prime}, P_{n}(x)=P_{n}\left(x^{\prime}\right)\right)$.

Let $\mathscr{R}_{1} \subseteq \mathscr{R}_{0}$ be the equivalence relation given by $\omega \mathscr{R}_{1} \omega^{\prime}$ iff $\omega \mathscr{R}_{0} \omega^{\prime}$ and for all $n$, $\omega_{n} \mathscr{S}_{n} \omega_{n}^{\prime}$. Let $\tilde{\mathscr{R}}_{1} \subseteq \tilde{\mathscr{R}}_{0}$ be given by $(\omega, t) \tilde{\mathscr{R}}_{1}\left(\omega^{\prime}, s\right)$ iff $\omega \mathscr{R}_{1} \omega^{\prime}$ and $t=s$.

Clearly $\Omega_{0} \times \mathbb{R} / \tilde{R}_{1}=\Omega_{0} / \mathscr{R}_{1} \times \mathbb{R}$ and $\left(\Omega_{0}, P\right) / \mathscr{R}_{1}=\prod_{n \geq 1}\left(\left(X_{n}, P_{n}\right) / \mathscr{S}_{n}\right)$.

Put $\left(X_{n}, P_{n}\right) / \mathscr{T}_{n}=\left(\Omega_{n}, \mu_{n}\right)$. Let $\mathscr{R}$ be the equivalence relation on $(\Omega, v)=$ $\prod_{n \geq 1}\left(\Omega_{n}, \mu_{n}\right)$ given by $x \mathscr{R} y$ iff there exists $k$ such that $x_{n}=y_{n}, n \geq k$. Let $\pi: \Omega_{0} \rightarrow \Omega$ be the projection. If $x \mathscr{R} y$, let $\omega, \omega^{\prime} \in \Omega_{0}$ be such that $\pi(\omega)=x, \pi\left(\omega^{\prime}\right)=y$ and $\omega \mathscr{R}_{0} \omega^{\prime}$. As $\mathscr{S}_{n}$ preserves $P_{n}$, we notice that

$$
\delta_{0}\left(\omega, \omega^{\prime}\right)=\prod_{n \geqslant 1} P_{n}\left(\omega_{n}\right) P_{n}\left(\omega_{n}^{\prime}\right)^{-1}
$$

does not depend on the choice of $\omega, \omega^{\prime}$. Put $\delta(x, y)=\delta_{0}\left(\omega, \omega^{\prime}\right)$. Then $\tilde{\mathscr{R}}$ is given by $(x, t) \tilde{R}(y, s)$ iff there exists $k \geq 1$ such that $x_{n}=y_{n}, n \geq k$ and $s=t-\log \delta(x, y)$.

(C) In $\S 3$, we have $\left(X_{n}, P_{n}\right)=\left(\{0,1\}, \alpha_{n}\right)^{\otimes K_{n}}$ where $\alpha_{n}(0)=1 /\left(1+\lambda_{n}\right), \alpha_{n}(1)=$ $\lambda_{n} /\left(1+\lambda_{n}\right)$. If $x=\left(x_{l}\right)_{l=1, \ldots, K_{n}} \in X_{n}$ put $k(x)=\#\left\{l \in\left\{1, \ldots, K_{n}\right\} ; x_{l}=1\right\}$. We have

$$
P_{n}(x)=\frac{\lambda_{n}^{k(x)}}{\left(1+\lambda_{n}\right)^{K_{n}}} \text {. }
$$

The equivalence relation $\mathscr{S}_{n}$ is here $x \mathscr{S}_{n} x^{\prime}$ iff $k(x)=k\left(x^{\prime}\right)$. The quotient of $X_{n}$ by $\mathscr{S}_{n}$ is $\left\{0, \ldots, K_{n}\right\}$ and the measure $\mu_{n}$ is given by

$$
\begin{aligned}
\mu_{n}(k) & =\sum_{x, k(x)=k} P_{n}(x) \\
& =(\#\{x, k(x)=k\}) \cdot \frac{\lambda_{n}^{k}}{\left(1+\lambda_{n}\right)^{K_{n}}}=\frac{K_{n} !}{\left(K_{n}-k\right) ! k !} \cdot \frac{\lambda_{n}^{k}}{\left(1+\lambda_{n}\right)^{K_{n}}} .
\end{aligned}
$$

It is also convenient in $\S 3$ to replace $\left\{0, \ldots, K_{n}\right\}$ by $\left\{-f_{n}, 1-f_{n}, \ldots, K_{n}-f_{n}\right\}$ considered as a subset of $\mathbb{Z}$. Note that if $\omega, \omega^{\prime} \in \Omega=\prod_{n \geq 1} \mathbb{Z}$ and if $\omega \mathscr{R} \omega^{\prime}$ then $\delta\left(\omega, \omega^{\prime}\right)=\prod_{n \geq 1} \lambda_{n}^{\omega_{n}-\omega_{n}^{\prime}}$ (if $\omega \mathscr{R} \omega^{\prime}$, all but finitely many $\omega_{n}-\omega_{n}^{\prime}$ are zero). 
(D) In $\$ 2$, we have $\left(X_{p, q}, P_{p, q}\right)=\left(\{0,1, \ldots, m, m+1\}, \quad \alpha_{p, q}\right)^{\otimes L_{p, q}}$ where $\alpha_{p, q}(0)=1 / \Lambda_{p, q}, \alpha_{p, q}(i)=\lambda_{p_{i}} / \Lambda_{p, q}, 1 \leq i \leq m$, and $\alpha_{p, q}(m+1)=\lambda_{q} / \Lambda_{p, q}$. For $x \in X_{p, q}$, $i=1, \ldots, m$, put $k_{i}(x)=\#\left\{l \in\left\{1, \ldots, L_{p, q}\right\} ; x_{l}=i\right]$ and $j(x)=\#\left\{l \in\left\{1, \ldots, L_{p, q}\right\}\right.$; $\left.x_{l}=m+1\right\}$. The equivalence $\mathscr{S}_{p, q}$ is given by $x \mathscr{Y}_{p, q} x^{\prime}$ iff $k_{i}(x)=k_{i}\left(x^{\prime}\right)$ for all $i$ and $j(x)=j\left(x^{\prime}\right)$. The quotient of $\left(X_{p, q}, P_{p, q}\right)$ by $\mathscr{S}_{p, q}$ is the space of $(k, j)$ considered in $\S 2$, namely $X_{p, q} / \mathscr{S}_{p, q}=\left\{(k, j) \in \mathbb{N}^{m} \times \mathbb{N} ;|k|+j \leq L_{p, q}\right\}$, and the quotient measure is the measure $\mu_{p, q}$ of $\S 2$.

We also consider

$$
\left(X_{p, q}^{\prime}, P_{p, q}^{\prime}\right)=\left(\{0,1, \ldots, m\}, \beta_{p}\right)^{\otimes K_{p, q}} \times\left(\{0,1\}, \gamma_{q}\right)^{\otimes J_{p, q}}
$$

where $\beta_{p}(i)=\lambda_{p_{i}} / \Lambda_{p}, \gamma_{q}(0)=1 /\left(1+\lambda_{q}\right), \gamma_{q}(1)=\lambda_{q} /\left(1+\lambda_{q}\right)$. An element $x \in X_{p, q}^{\prime}$ is of the form $(y, z), y=\left(y_{l}\right)_{l=1, \ldots, K_{p, q}}, y_{l} \in\{0,1, \ldots, m\}, z=\left(z_{p}\right)_{l=1, \ldots, J_{p, q}}, z_{l} \in\{0,1\}$. If $x \in X_{p, q}^{\prime}$ put $k_{i}(x)=\#\left\{l \in\left\{1, \ldots, K_{p, q}\right\} ; y_{l}=i\right\}$ and $j(x)=\#\left\{l \in\left\{1, \ldots, J_{p, q}\right\} ; z_{l}=1\right\}$.

The equivalence relation $\mathscr{S}_{p, q}^{\prime}$ is given by $x \mathscr{P}_{p, q}^{\prime} x^{\prime}$ iff $k_{i}(x)=k_{i}\left(x^{\prime}\right)$ for all $i$ and $j(x)=j\left(x^{\prime}\right)$. The quotient of $\left(X_{p, q}^{\prime}, P_{p, q}^{\prime}\right)$ by $\mathscr{P}_{p, q}^{\prime}$ is the space $\left\{(k, j) \in \mathbb{N}^{m} \times \mathbb{N},|k| \leq K_{p, q}\right.$, $\left.j \leq J_{p, q}\right\}$ and the measure $\mu_{p, q}^{\prime}$ of $\S 2$.

\section{REFERENCES}

[1] H. Araki \& E. J. Woods. A classification of factors. Publ Res. Inst. Math. Sci. Ser. A 4 (1968), 51-130.

[2] A. Connes. Une classification des facteurs de type III. Ann Sci. E.N.S. 4 eme Série 6 (1973), 133-252.

[3] A. Connes. On the hierarchy of W. Krieger. Illinois J. Math. 19, (1975), 428-432.

[4] A. Connes. Sur la Théorie non-commutative de l'Intégration. Lecture Notes in Math. no 725. Springer Verlag, 1979.

[5] A. Connes \& M. Takesaki. The flow of weights on factors of type III. Tôhoku Math. J. 29 (1977), 473-575.

[6] A. Connes, J. Feldman \& B. Weiss. An amenable equivalence relation is generated by a single transformation. Ergod. Th. \& Dynam. Sys. 1 (1981), 431-450.

[7] N. Draper \& W. Lawrence. Probability: an Introductory Course. Markham: Chicago, 1970.

[8] P. Hahn. Regular representations of measure groupoids. Trans. Amer. Math. Soc. 242 (1978), 35-72.

[9] P. Halmos. Measure Theory. van Nostrand, 1950.

[10] T. Hamachi \& M. Osikawa. Ergodic Groups of Automorphisms and Krieger's Theorems. Seminar on Math. Sci. Keio Univ. 3 (1981).

[11] E. Hewitt \& K. Stromberg. Real and Abstract Analysis. Springer Verlag: New York, 1969.

[12] W. Krieger. On ergodic flows and the isomorphism of factors. Math. Ann. 223 (1976), 19-70.

[13] J. L. Sauvageot. Image d'un homomorphisme et flot des poids d'une relation d'équivalence mesurée. C.R. Acad. Sci. Paris, Sér A. 282 (1976), 619-621.

[14] C. Sutherland. Notes on orbit equivalence: Krieger's Theorem, Lecture Note series No. 23, Univ. i Oslo, 1976. Preprint.

[15] E. J. Woods. The classification of factors is not smooth. Can. J. Math. 25 (1973), 96-102.

[16] E. J. Woods. ITPFI factors-A survey. In Proc. of Symposia in Pure Mathematics 38 (1982), Part $2,25-41$. 